\title{
An SDRE Based Estimator Approach for HIV Feedback Control
}

\author{
H. T. Banks, Hee-Dae Kwonł J. A. Toivanen*, and H. T. Tran*‡
}

April 24, 2005

\begin{abstract}
We consider optimal dynamic multidrug therapies for human immunodeficiency virus (HIV) type 1 infection. In this context we describe an optimal tracking problem attempting to drive the states of the system to a stationary state in which the viral load is low and the immune response is strong. We consider optimal feedback control with full state as well as with partial state measurements. In the case of partial state measurement, a state estimator is constructed based on viral load and T-cell count measurements. We demonstrate by numerical simulations that by anticipation of and response to the disease progression, the dynamic multidrug strategy reduces the viral load, increases the CD4+ T-cell count and improves the immune response.
\end{abstract}

${ }^{*}$ Center for Research in Scientific Computation, Box 8205, North Carolina State University, Raleigh, NC 27695-8205.

${ }^{\dagger}$ Department of Mathematics, Inha University, Incheon, South Korea. Email: hdkwon@inha.ac.kr.

${ }^{\ddagger}$ Author for correspondence. E-mail: tran@math.ncsu.edu 


\section{Introduction}

During the last two decades medical treatment for human immunodeficiency virus (HIV) has greatly improved. Typically therapy can prolong time to onset of acquired immune deficiency syndrome (AIDS) for tens of years. The prevailing medical practice is to prescribe highly active antiretroviral therapy (HAART) which can reduce viral load and maintain high CD4+ T-cell counts. This therapy involves combinations of three or more drugs that are called "cocktails". However, in spite of the success of HAART, some patients develop resistance to one or more of the drugs in long term use. In these cases, it is necessary to change the composition of HAART. In addition, there may be severe side effects from the medication. Moreover, in developing countries, the expense of HAART is often prohibitively high. Motivated by these and other reasons, the search for alternative treatments is very active. In this paper we study dynamic multidrug therapies that can lead to long-term control of HIV by the immune response system after discontinuation of drug treatment.

HIV infects CD4+ T-cells (a fundamental component of the human immune response system) and other target cells, hijacking their replication mechanisms. The infected cells then produce a large number of copies of the virus. Currently the two most important categories of anti-HIV drugs are reverse transcriptase inhibitors (RTIs) and protease inhibitors (PIs). A typical HAART cocktail consists of one or more RTIs and a PI. The reverse transcriptase inhibitors prevent HIV from infecting cells by blocking the integration of the viral code into the target cells. Protease inhibitors interfere with the replication of viruses by infected cells. Virions may still be produced, but they are generally non infectious; that is, they are not capable of infecting new target cells. In practice, RTIs cannot completely block the virus integration of the DNA in target cells. Also, some infectious virions are produced under PI medication. Every drug has a maximum efficacy which depends on many factors such as, for example, viral strains present. One might expect that the effectiveness of HIV therapy could be improved by developing dynamic multidrug strategies, where the combination of drugs given to HIV patients changes over time in response to the individual's disease progression.

A number of different mathematical models based on systems of differential equations have been developed, see for example [24]. Some of these models used to design dynamical drug treatments are presented in $[1,8,24,25,29,30,31]$. In the long term pathogenesis of HIV an immune response can play an important role. However, the models in [24, 25] do not contain immune response while the authors in $[1,8,29,30,31]$ do consider the immune response. Since immune mechanisms responding to HIV are not yet very well understood, various immune response models have been proposed in the literature. In this study, we employ a model based on the models considered in $[1,2]$ which contain an immune effector component.

Optimal treatment of HIV infection using a control theoretic approach is the subject of substantial research activity. The papers $[1,3,10,13,14,26]$ consider only RTI medication while the papers $[20,22]$ consider only PIs. In [33, 34, 35] all effects of a HAART medication are combined and represented by one control variable in the model. In $[2,11,17,21,28]$ dynamical multidrug therapies based on RTIs and PIs are designed. In these proposed

therapies the dosage of both medications can change independently of each other and can either be continuous or on-off types. Studies of continuously varying medical therapies have been more common, see, e.g., [1, 3, 9, 10, 11, 13, 14, 17, 18, 20, 22, 28, 30]. More recently, 
the on-off type of treatment, which is also known as a structured treatment interruption (STI), has attracted a lot of attention in the medical literature (see for example, $[1,2,4]$ and the references therein). A primary argument for use of STI therapy instead of continuously varying dosage is that one might lower the risk of HIV mutating to strains which are resistant to the current medication regimen. Recent results on structured treatment interruption schedules including optimal treatments are presented in $[1,2,21,34,35]$. In this paper we consider optimal feedback treatment of HIV infection by continuously varying dosages of RTIs and PIs in a nonlinear model including an immune response.

There are a number of control techniques that can be utilized to design dynamical therapies for HIV. Open loop control has been employed in $[1,2,10,13,14,18,20,21,26,28]$ and feedback control has been used in $[3,9,11,26,33,34,35]$. The papers $[3,9,33]$ consider the feedback control based on partial measurements. An estimator is employed to construct the control in [3] while in [33] this possibility is only briefly mentioned. A linear quadratic regulator (LQR) control based on Riccati equations is studied in [11].

For linear systems, LQR is a well-known and accepted methodology for the synthesis of control laws. However, most mathematical models for biological systems, including the HIV dynamics with immune response as studied in this paper, are nonlinear. One of the promising and emerging methodologies for designing nonlinear controllers is the state dependent Riccati equation (SDRE) approach in the context of nonlinear regulator problems (see for example, $[6,7,16,23]$ ). In essence, the SDRE method is a systematic way of designing nonlinear feedback controllers by factoring the state dependent nonlinearity of the state equations as a product of a state dependent matrix with the state vector. That is, by using direct parameterization the nonlinear system is brought to a linear structure with state dependent coefficient matrices. This parametrization is however not unique and thus some flexibility in design is permissible. The state feedback control law is then given in terms of the solution of a state dependent Riccati equation. As shown in [7], the SDRE method is a powerful approach that is readily applicable to the nonlinear tracking and nonlinear state estimation problems, since it is closely related to the algebraic Riccati equation-based method used to find the feedback controls in the linear cases.

While the SDRE method has been applied earlier to mostly engineering type problems such as flight dynamics simulation [7] and chemical vapor deposition [5], the idea of using SDRE for combined drug/immune response control of HIV infection as presented here is new. In addition, we propose, in this paper, a more systematic approach to parametrization of the nonlinear system as a linear structure with state dependent coefficient matrices. In our approach, the state dependent matrix is the Jacobian of the nonlinear system dynamics. Our parametrization choice together with the proposed time discretization method imply that the state dependent coefficient matrix is in fact the exact local linearization of the nonlinear state dependent system dynamics at the current state of the system.

The outline of the paper is as follows. We begin in Section 2 with a description of a rather complex HIV model. An optimal quadratic tracking problem is formulated in Section 3 to drive the state of the system to a stationary state which we call the "healthy" state (it has low viral load and high CD4+ T-cell count). Section 4 contains a direct factorization of the nonlinear system into a linear form with state dependent coefficient matrices. By then mimicking standard LQR formulation for linear systems, a suboptimal feedback control is derived in Section 5. Sections 6 and 7 present the formulation of the state estimator for more realistic control problems where only partial state measurements are available for feedback. In Section 8 and 9 we summarize the numerical procedure 
for the synthesis of the suboptimal feedback controls. Numerical simulation results are summarized in Section 10 and concluding remarks are presented in Section 11.

\section{HIV Model}

A modification of the mathematical model as presented in $[1,2]$ for the pathogenesis of HIV is adopted for this paper. We include an additional state variable for non infectious virus $[12,24]$. The model is essentially based on the two target model proposed by Callaway and Perelson in [12] without making distinction between short and long lived infected target cells. In addition, it contains an immune response component similar to the one described by Bonhoeffer, et al., in [8] with a Michaelis-Menten type saturation nonlinearity. Our model captures many of the observed behavioral properties of HIV dynamics [8, 12] and, furthermore, its mathematical properties are well-suited for designing multidrug therapies using control theoretic approaches $[1,2]$. The purpose of adding non infectious virus to the model is to reflect what is actually being measured in clinical data. The viral load measurements cannot differentiate between infectious and non infectious virus, detecting only the total amount of virus. Hence, in order to construct a state estimator (for developing dynamic multidrug therapy based on the disease progression) using such measurements, we need to model also the non infectious virus population. It should be noted that the inclusion of this additional state does not effect the dynamics of the other state variables.

The dynamics of our HIV model are described by the set of ordinary differential equations:

$$
\begin{aligned}
\dot{T}_{1} & =\lambda_{1}-d_{1} T_{1}-\left(1-\epsilon_{1}\right) k_{1} V_{I} T_{1} \\
\dot{T}_{2} & =\lambda_{2}-d_{2} T_{2}-\left(1-f \epsilon_{1}\right) k_{2} V_{I} T_{2} \\
\dot{T}_{1}^{*} & =\left(1-\epsilon_{1}\right) k_{1} V_{I} T_{1}-\delta T_{1}^{*}-m_{1} E T_{1}^{*} \\
\dot{T}_{2}^{*} & =\left(1-f \epsilon_{1}\right) k_{2} V_{I} T_{2}-\delta T_{2}^{*}-m_{2} E T_{2}^{*} \\
\dot{V}_{I} & =\left(1-\epsilon_{2}\right) N_{T} \delta\left(T_{1}^{*}+T_{2}^{*}\right)-\left[c+\left(1-\epsilon_{1}\right) \rho_{1} k_{1} T_{1}+\left(1-f \epsilon_{1}\right) \rho_{2} k_{2} T_{2}\right] V_{I} \\
\dot{V}_{N I} & =\epsilon_{2} N_{T} \delta\left(T_{1}^{*}+T_{2}^{*}\right)-c V_{N I} \\
\dot{E} & =\lambda_{E}+b_{E} \frac{T_{1}^{*}+T_{2}^{*}}{T_{1}^{*}+T_{2}^{*}+K_{b}} E-d_{E} \frac{T_{1}^{*}+T_{2}^{*}}{T_{1}^{*}+T_{2}^{*}+K_{d}} E-\delta_{E} E .
\end{aligned}
$$

In the model (1), the state variables are: $T_{1}$, the uninfected CD4+ T-cells; $T_{2}$, the uninfected target cells of second kind; $T_{1}^{*}$, the infected T-cells; $T_{2}^{*}$, the infected target cells of second kind; $\dot{V}_{I}$, the infectious virus; $\dot{V}_{N I}$, the non infectious virus; and $E$, the immune effectors. The controllers $\epsilon_{1}$ and $\epsilon_{2}$ represent the RTI and PI "efficacies", respectively. We do not give precise biological definitions for the target cells of second kind and the immune effectors. They could, for example, be related to macrophages and cytotoxic Tlymphocytes, respectively. For a more detailed description of the variables and rationale for the model (1) we refer the reader to the articles $[1,2]$. Table 1 contains the values of parameters, which are the same as those used in $[1,2]$. The only difference is that we use $\mathrm{mm}^{3}$ (cubic millimeter) as our unit volume instead of ml (milliliter).

In order to simplify our subsequent discussions, we introduce the notation $x$ and $u$ to 


\begin{tabular}{ccc|ccc} 
parameter & value & unit & parameter & value & unit \\
\hline$\lambda_{1}$ & 10.0 & $\frac{\text { cells }}{\mathrm{mm}^{3} \cdot \text { day }}$ & $\lambda_{2}$ & $31.98 \times 10^{-3}$ & $\frac{\text { cells }}{\mathrm{mm}^{3} \cdot \text { day }}$ \\
$d_{1}$ & 0.01 & $\frac{1}{\text { day }}$ & $d_{2}$ & 0.01 & $\frac{1}{\text { day }}$ \\
$k_{1}$ & $8.0 \times 10^{-4}$ & $\frac{\mathrm{mm}^{3}}{\text { virions·day }}$ & $k_{2}$ & 0.1 & $\frac{\mathrm{mm}^{3}}{\text { virions.day }}$ \\
$m_{1}$ & 0.01 & $\frac{\mathrm{mm}^{3}}{\text { cells·day }}$ & $m_{2}$ & 0.01 & $\frac{\text { mm }}{\text { cells.day }}$ \\
$\rho_{1}$ & 1 & $\frac{\text { virions }}{\text { cells }}$ & $\rho_{2}$ & 1 & $\frac{\text { virions }}{\text { cells }}$ \\
$\delta$ & 0.7 & $\frac{1}{\text { day }}$ & $c$ & 13.0 & $\frac{1}{\text { day }}$ \\
$f$ & 0.34 & - & $N_{T}$ & 100.0 & $\frac{\text { virions }}{\text { cells }}$ \\
$\lambda_{E}$ & $1.0 \times 10^{-3}$ & $\frac{\text { cells }}{\mathrm{mm}^{3} \cdot \text { day }}$ & $\delta_{E}$ & 0.1 & $\frac{1}{\text { day }}$ \\
$b_{E}$ & 0.3 & $\frac{1}{\text { day }}$ & $d_{E}$ & 0.25 & $\frac{1}{\text { day }}$ \\
$K_{b}$ & 0.1 & $\frac{\text { cells }}{\mathrm{mm}^{3}}$ & $K_{d}$ & 0.5 & $\frac{\text { cells }}{\mathrm{mm}^{3}}$
\end{tabular}

Table 1: The values of the parameters in the HIV model.

denote the state and control vectors, respectively. Thus we define

$$
x=\left(\begin{array}{c}
T_{1} \\
T_{2} \\
T_{1}^{*} \\
T_{2}^{*} \\
V_{I} \\
V_{N I} \\
E
\end{array}\right) \quad \text { and } \quad u=\left(\begin{array}{c}
\epsilon_{1} \\
\epsilon_{2}
\end{array}\right) .
$$

With the above notation, the HIV model (1) can be expressed in the generic form

$$
\dot{x}=f(x)+B(x) u,
$$

where the precise representation for $f(x)$ and $B(x)$ will be described later in Section 4 .

For feedback control we need current knowledge on the state of the system. In our effort here we assume that partial state observations $\left(T_{1}+T_{1}^{*}, V_{I}+V_{N I}\right)$ are available. This is representative of the type of clinical data widely discussed in the literature (see for example, [1]). Hence, the output or observation takes the form

$$
z=\left(\begin{array}{l}
z_{1} \\
z_{2}
\end{array}\right)=\left(\begin{array}{ccccccc}
1 & 0 & 1 & 0 & 0 & 0 & 0 \\
0 & 0 & 0 & 0 & 1 & 1 & 0
\end{array}\right) x=C x
$$

where $z_{1}$ and $z_{2}$ represent the total CD4+ counts and the total viral loads, respectively.

\section{An Optimal Tracking Problem Formulation}

With the parameters given in Table 1 and the assumption of no medication $\left(\epsilon_{1}=\epsilon_{2}=0\right)$, the model exhibits several steady states. These are described and analyzed in $[1,2]$ with the non infectious virus $V_{N I}$ state always being zero in these steady states. We are particularly interested in the so-called "healthy" steady state given by

$$
\begin{array}{ll}
T_{1}=967.839 \frac{\text { cells }}{\mathrm{mm}^{3}}, & T_{2}=0.621 \frac{\text { cells }}{\mathrm{mm}^{3}}, \quad T_{1}^{*}=0.076 \frac{\text { cells }}{\mathrm{mm}^{3}}, \quad T_{2}^{*}=0.006 \frac{\text { cells }}{\mathrm{mm}^{3}}, \\
V_{I}=0.415 \frac{\text { virions }}{\mathrm{mm}^{3}}, & V_{N I}=0.0 \frac{\text { virions }}{\mathrm{mm}^{3}}, \quad \text { and } \quad E=353.108 \frac{\text { cells }}{\mathrm{mm}^{3}},
\end{array}
$$


which was also shown to be locally asymptotically stable. This means that after a sufficiently small perturbation from (4), the trajectory of the state $x$ returns to the stable equilibrium (4). It is noted that this stable equilibrium exhibits a strong immune response, low viral load and reasonably large target cell $\left(T_{1}\right)$ counts.

Highly active antiretroviral therapy (HAART) has proven to be very effective at reducing the viral load to below detectable levels. However, sustainable suppression has proven to be difficult using HAART because patients taking these drugs experience adverse side effects that make adherence to therapy very difficult. In this paper we adopt a control theoretic approach to find a suboptimal treatment strategy that can lead to high immune effector levels and subsequent control of viral load without the need for further drug therapy.

We formulate the problem of finding an effective multidrug therapy as a tracking problem. To this end, we define the objective functional

$$
J(x, u)=\frac{1}{2} \int_{0}^{\infty}\left\{\left(V_{I}-0.415\right)^{2}+10(E-353.108)^{2}+\left(\epsilon_{1} / \epsilon_{1}^{\max }\right)^{2}+\left(\epsilon_{2} / \epsilon_{2}^{\max }\right)^{2}\right\} d t,
$$

where $V_{I}$ is the number of free virus and $E$ represents the immune response. The control variable $\epsilon_{1}$, where $0 \leq \epsilon_{1} \leq \epsilon_{1}^{\max }$, denotes the "efficacy" of the reverse transcriptase inhibitor. Similarly, the control variable $\epsilon_{2}, 0 \leq \epsilon_{2} \leq \epsilon_{2}^{\max }$, represents the "efficacy" of the protease inhibitor. We note that throughout we use the somewhat nonstandard terminology "efficacy" interchangeably with the control level for the two drugs. The weights in (5) have been determined a priori through a series of numerical experiments. The feedback control algorithm proposed in Section 5 is not particularly sensitive to the choice of these weights.

We denote the control vector $u$ by

$$
u=\left(\begin{array}{c}
\epsilon_{1} \\
\epsilon_{2}
\end{array}\right)
$$

We also introduce the following two vectors

$$
\check{u}=\left(\begin{array}{l}
0 \\
0
\end{array}\right) \quad \text { and } \quad \hat{u}=\left(\begin{array}{c}
\epsilon_{1}^{\max } \\
\epsilon_{2}^{\max }
\end{array}\right) .
$$

The optimal tracking control problem is to find a dynamic multidrug therapy $u(t)$ satisfying

$$
\min _{\check{u} \leq u(t) \leq \hat{u}} J(x(t), u(t))
$$

subject to the state equation given by (2) with initial condition $x(0)=x_{0}$. We could, of course, consider the restricted class of scaler controllers $u$ where $\epsilon_{i}(t)=\hat{\epsilon}_{i} u(t)$. While this is the more usual treatment protocol in clinical practice, we are interested in investigation here of the more flexible scenario where the RTI and PI levels can vary independently. We also note that although we formulate a continuous feedback, current clinical practice involves discrete observations and hence our approach, if implemented, would have to be approximated by some type of interpolated estimate of the observed states (see especially the computational examples below where impractical observation sampling is used).

We note that our mathematical model for HIV dynamics (2) is nonlinear. One of the highly promising and emerging techniques for designing nonlinear feedback controllers is 
the state-dependent Riccati equation (SDRE) approach in the context of the nonlinear regulator problem. This technique essentially uses direct parametrization to rewrite the nonlinear state function $f(x)$ in $(2)$ as a product of a state-dependent coefficient matrix with the state vector. This parametrization is, of course, not unique and one obvious factorization is given in terms of the local linearization of the state equation.

\section{A Local Linearization of the State Equation}

To design a nonlinear feedback controller using the SDRE methodology, one usually first rewrites the nonlinear dynamics (2) in the state-dependent coefficient form $f(x)=A(x) x$. Underlying this form is the tacit assumption that $f(0)=0$. Our HIV model (1) does not satisfy this condition. For this reason we divide $f(x)$ into two parts

$$
f(x)-a \quad \text { and } \quad a,
$$

where $a$ is chosen in such a way that $f(0)-a=0$. The simplest choice is $a=f(0)$. However, instead of using this simple choice we let $a$ depend on $x$ and we denote this dependence by $a(x)$. The choice of $a(x)$ will become clear below.

We thus rewrite the state equation (2) as

$$
\dot{x}=A(x) x+a(x)+B(x) u .
$$

That is, $f(x)$ is parameterized as

$$
f(x)=A(x) x+a(x) .
$$

It has been noted $[7,16,23]$ that the choice of $A(x)$ in systems where $f(0)=0$ is not unique and the same also holds for our generalized formulation. However, for this formulation a natural choice of the matrix $A(x)$ is the Jacobian of $f(x)$. That is,

$$
A(x)=\frac{\partial f(x)}{\partial x} \quad \text { and } \quad a(x)=f(x)-A(x) x .
$$

For our particular HIV model (1) the Jacobian is given by

$$
A(x)=\left(\begin{array}{ccccccc}
-d_{1}-k_{1} V_{I} & 0 & 0 & 0 & -k_{1} T_{1} & 0 & 0 \\
0 & -d_{2}-k_{2} V_{I} & 0 & 0 & -k_{2} T_{2} & 0 & 0 \\
k_{1} V_{I} & 0 & -\delta-m_{1} E & 0 & k_{1} T_{1} & 0 & -m_{1} T_{1}^{*} \\
0 & k_{2} V_{I} & 0 & -\delta-m_{2} E & k_{2} T_{2} & 0 & -m_{2} T_{2}^{*} \\
-\rho_{1} k_{1} V_{I} & -\rho_{2} k_{2} V_{I} & N_{T} \delta & N_{T} \delta & A_{55} & 0 & 0 \\
0 & 0 & 0 & 0 & 0 & -c & 0 \\
0 & 0 & A_{73} & A_{74} & 0 & 0 & A_{77}
\end{array}\right),
$$

where

$$
\begin{gathered}
A_{55}=-c-\rho_{1} k_{1} T_{1}-\rho_{2} k_{2} T_{2} \\
A_{73}=A_{74}=\frac{b_{E} K_{b} E}{\left(T_{1}^{*}+T_{2}^{*}+K_{b}\right)^{2}}-\frac{d_{E} K_{d} E}{\left(T_{1}^{*}+T_{2}^{*}+K_{d}\right)^{2}}
\end{gathered}
$$

and

$$
A_{77}=\left(\frac{b_{E}}{T_{1}^{*}+T_{2}^{*}+K_{b}}-\frac{d_{E}}{T_{1}^{*}+T_{2}^{*}+K_{d}}\right)\left(T_{1}^{*}+T_{2}^{*}\right)-\delta_{E} .
$$


Finally, the matrix $B(x)$ in (7) is given by

$$
B(x)=\left(\begin{array}{cc}
k_{1} V_{I} T_{1} & 0 \\
f k_{2} V_{I} T_{2} & 0 \\
-k_{1} V_{I} T_{1} & 0 \\
-f k_{2} V_{I} T_{2} & 0 \\
\rho_{1} k_{1} V_{I} T_{1}+f \rho_{2} k_{2} V_{I} T_{2} & -N_{T} \delta\left(T_{1}^{*}+T_{2}^{*}\right) \\
0 & N_{T} \delta\left(T_{1}^{*}+T_{2}^{*}\right) \\
0 & 0
\end{array}\right) .
$$

\section{Optimal Feedback Controllers}

In this section we formally derive the nonlinear feedback controllers for the optimal tracking problem described in Section 3. We first will assume that all state variables are available for feedback. Our approach is to use the SDRE technique and mimic the standard linear quadratic regulator formulation for linear systems.

We begin by rewriting the objective functional in (5) in the generic form

$$
J(x, u)=\frac{1}{2} \int_{0}^{\infty}\left[(x-y)^{T} Q(x-y)+u^{T} R u\right] d t
$$

where the dynamic tracking variable $y$ represents the stable equilibrium state (4).

The Hamiltonian for our optimal control problem is given by

$$
\begin{aligned}
\mathcal{H}(x, u, p) & =\frac{1}{2}(x-y)^{T} Q(x-y)+\frac{1}{2} u^{T} R u+p^{T}(A(x) x+a(x)+B(x) u) \\
& -\check{w}^{T}(u-\check{u})-\hat{w}^{T}(\hat{u}-u),
\end{aligned}
$$

where the penalty multiplier vectors $\check{w}$ and $\hat{w}$, are introduced to account for the constraints on the control variable $u$, are non negative and satisfy the conditions

$$
\check{w}^{T}(u-\check{u})=\hat{w}^{T}(\hat{u}-u)=0
$$

at the optimal control. From the Hamiltonian, the necessary conditions for optimality are found to be

$$
\begin{aligned}
& \dot{x}=A(x) x+a(x)+B(x) u=\frac{\partial \mathcal{H}}{\partial p}, \\
& \dot{p}=-Q(x-y)-\left[\frac{d(A(x) x)}{d x}\right]^{T} p-\left[\frac{d(a(x))}{d x}\right]^{T} p-\left[\frac{d(B(x) u)}{d x}\right]^{T} p=-\frac{\partial \mathcal{H}}{\partial x}, \\
& 0=R u+B^{T}(x) p-\check{w}+\hat{w}=\frac{\partial \mathcal{H}}{\partial u} .
\end{aligned}
$$

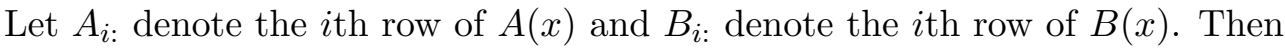

$$
\begin{aligned}
\frac{d(A(x) x)}{d x} & =A(x)+\frac{d(A(x))}{d x} x \\
& =A(x)+\left[\begin{array}{ccc}
\frac{d A_{1:}}{d x_{1}} x & \ldots & \frac{d A_{1:}}{d x_{n}} x \\
\vdots & \ddots & \vdots \\
\frac{d A_{n:}}{d x_{1}} x & \ldots & \frac{d A_{n:}}{d x_{n}} x
\end{array}\right]
\end{aligned}
$$


and

$$
\frac{d(B(x) u)}{d x}=\left[\begin{array}{ccc}
\frac{d B_{1:}}{d x_{1}} u & \ldots & \frac{d B_{1:}}{d x_{n}} u \\
\vdots & \ddots & \vdots \\
\frac{d B_{n:}}{d x_{1}} u & \ldots & \frac{d B_{n:}}{d x_{n}} u
\end{array}\right]
$$

The last equation of (9) leads to the optimal control

$$
u=-R^{-1}\left(B^{T}(x) p-\check{w}+\hat{w}\right) .
$$

Mimicking the tracking problem for linear systems, we assume that the adjoint state has the form

$$
p=\Pi(x) x+b(x),
$$

where $\Pi$ is a matrix, $b$ is a vector and both are state dependent. To find $\Pi(x)$ and $b(x)$, we differentiate (12) with respect to time along a trajectory and substitute the optimality condition (9) for $\dot{p}$ to obtain

$$
-Q(x-y)-\left[\frac{d(A(x) x)}{d x}\right]^{T} p-\left[\frac{d(a(x))}{d x}\right]^{T} p-\left[\frac{d(B(x) u)}{d x}\right]^{T} p=\dot{\Pi} x+\Pi \dot{x}+\dot{b},
$$

where we use the notation

$$
\dot{\Pi}(x)=\sum_{i=1}^{n} \Pi_{x_{i}}(x) \dot{x}_{i}(t) .
$$

A similar definition also holds for $\dot{b}$. Substituting the expressions for $p, \dot{x}$, and $u$ and rearranging terms, we find

$$
\begin{aligned}
& {\left[\left(\dot{\Pi}(x)+\left[\frac{d(A(x))}{d x} x\right]^{T} \Pi(x)+\left[\frac{d(B(x) u)}{d x}\right]^{T} \Pi(x)+\left[\frac{d(a(x))}{d x}\right]^{T} \Pi(x)\right)\right.} \\
& \left.+\left(\Pi(x) A(x)+A^{T}(x) \Pi(x)-\Pi(x) B(x) R^{-1} B^{T}(x) \Pi(x)+Q\right)\right] x \\
& +\dot{b}-Q y+\Pi(x) a(x)-\Pi(x) B(x) R^{-1}(-\check{w}+\hat{w}) \\
& +\left(A^{T}(x)-\Pi(x) B(x) R^{-1} B^{T}(x)+\left[\frac{d(A(x))}{d x} x\right]^{T}+\left[\frac{d(B(x) u)}{d x}\right]^{T}+\left[\frac{d(a(x))}{d x}\right]^{T}\right) b=0 .
\end{aligned}
$$

If we approximate (see [7]) by assuming that the derivative terms

$$
\frac{d(A(x))}{d x}, \frac{d(B(x) u)}{d x}, \frac{d(a(x))}{d x}
$$

are small as well as that $\Pi$ and $b$ are stationary, it follows that the suboptimal solution to the optimal tracking problem is given by

$$
u=\min (\max (\tilde{u}, \check{u}), \hat{u}),
$$

where minimum and maximum are taken component wise and

$$
\tilde{u}=-R^{-1} B^{T}(x)(\Pi(x) x+\tilde{b}(x)) .
$$


It should be emphasized that the above approximate feedback law for dynamic multidrug treatment is only suboptimal since the formula is only valid if both controls don't violate the constraints or violate the constraint simultaneously. However, our numerical studies suggest that the feedback law works reasonably well even when these conditions are not met. The state dependent function $\tilde{b}(x)$ is given by the expression

$$
\tilde{b}=\left(A^{T}(x)-\Pi(x) B(x) R^{-1} B^{T}(x)\right)^{-1}(Q y-\Pi(x) a(x)),
$$

where $\Pi(x)$ satisfies the state dependent Riccati equation (SDRE)

$$
A^{T}(x) \Pi(x)+\Pi(x) A(x)-\Pi(x) B(x) R^{-1} B^{T}(x) \Pi(x)+Q=0 .
$$

One approach involves obtaining the solution to SDRE is by use of symbolic software packages such as Macsyma or Mathematica. However, once the nonlinear dynamics of the system become complex, one has to rely on numerical approximation techniques to obtain its solution. In Sections 8 and 9, we will discuss the numerical procedure that we used to obtain the solution of the SDRE.

\section{Compensator Design}

For the synthesis of the nonlinear feedback control law derived in Section 5, full knowledge of all the state variables is required. However, in many problems of practical interest, only partial measurements of the state are available. In this section, we consider the problem of designing a state estimator to be used in conjunction with the nonlinear feedback control laws described earlier. We recall that the dynamical system and the observation are given by

$$
\begin{aligned}
\dot{x} & =f(x)+B(x) u \\
z & =C x,
\end{aligned}
$$

where the output matrix $C$ is defined by (3).

As in the linear problem, we design the state estimator to be of the form

$$
\dot{x_{e}}=f_{e}\left(x_{e}\right)+F\left(C x, x_{e}\right),
$$

where the functions $f_{e}$ and $F$ are to be specified later. It should be emphasized that the rationale behind the state estimator is that it is indeed the state estimator $x_{e}$ (and not the state $x$ ) that is to be used in the nonlinear feedback control laws given by equations (15)(16). Defining the error function between the state and the state estimator as $e=x-x_{e}$ and taking its derivative, we obtain

$$
\dot{e}=f(x)+B(x) u-f_{e}\left(x_{e}\right)-F\left(C x, x_{e}\right) .
$$

If we choose $f_{e}\left(x_{e}\right)=f\left(x_{e}\right)+B\left(x_{e}\right) u-F\left(C x_{e}, x_{e}\right)$, the equation for the derivative of the error function becomes

$$
\dot{e}=\left(f(x)+B(x) u-F\left(C x, x_{e}\right)\right)-\left(f\left(x_{e}\right)+B\left(x_{e}\right) u-F\left(C x_{e}, x_{e}\right)\right) .
$$

We next parameterize the nonlinear function $f(x)$ as

$$
f(x)=A(x) x+a(x),
$$


and we choose $F$ to be the product

$$
F\left(C x, x_{e}\right)=L\left(x_{e}\right) C x
$$

The derivative of the error then becomes

$$
\begin{aligned}
\dot{e} & =\left(A(x) x+a(x)+B(x) u-L\left(x_{e}\right) C x\right)-\left(A\left(x_{e}\right) x_{e}+a\left(x_{e}\right)+B\left(x_{e}\right) u-L\left(x_{e}\right) C x_{e}\right) \\
& =\left(A\left(x_{e}\right)-L\left(x_{e}\right) C\right) e+\left(B(x)-B\left(x_{e}\right)\right) u+A(x) x-A\left(x_{e}\right) x+a(x)-a\left(x_{e}\right) \\
& =\left(A\left(x_{e}\right)-L\left(x_{e}\right) C\right) e+\left(\sum_{i} \frac{\partial B \cdot i\left(x_{e}\right)}{\partial x} u_{i}\right) e+\mathcal{O}\left(\|e\|^{2}\right)+f(x)-f\left(x_{e}\right)-A\left(x_{e}\right) e \\
& =\left(A\left(x_{e}\right)+\sum_{i} \frac{\partial B \cdot i\left(x_{e}\right)}{\partial x_{e}} u_{i}-L\left(x_{e}\right) C\right) e+\mathcal{O}\left(\|e\|^{2}\right),
\end{aligned}
$$

where we have used the Taylor series

$$
B(x) u=B\left(x_{e}\right) u+\left(\sum_{i} \frac{\partial B \cdot i\left(x_{e}\right)}{\partial x_{e}} u_{i}\right) e+\mathcal{O}\left(\|e\|^{2}\right)
$$

and

$$
f(x)=f\left(x_{e}\right)+A\left(x_{e}\right) e+\mathcal{O}\left(\|e\|^{2}\right) .
$$

The subscript $\cdot i$ refers to the $i$ th column of the associated matrix. In order to simplify the notation, we define

$$
\hat{A}\left(x_{e}\right)=A\left(x_{e}\right)+\sum_{i} \frac{\partial B_{\cdot i}\left(x_{e}\right)}{\partial x_{e}} u_{i}
$$

Since the goal of designing a state estimator is that it approximates the true state of the system closely, the error $e$ should be small. Therefore, from equation (17), the term $\left(\hat{A}\left(x_{e}\right)-L\left(x_{e}\right) C\right) e$ dominates $\mathcal{O}\left(\|e\|^{2}\right)$. For this reason we neglect the term $\mathcal{O}\left(\|e\|^{2}\right)$ from the rest of our discussion. Now, if we choose $L\left(x_{e}\right)$ so that the eigenvalues of $\hat{A}\left(x_{e}\right)-$ $L\left(x_{e}\right) C$ have negative real parts, the estimation error $e$ will converge to zero asymptotically as $t \rightarrow \infty$ (hence, $x_{e}$ will approach $x$ ). Since $\hat{A}\left(x_{e}\right)-L\left(x_{e}\right) C$ and $\hat{A}^{T}\left(x_{e}\right)-C^{T} L^{T}\left(x_{e}\right)$ have the same eigenvalues, we can design the compensator gain $L\left(x_{e}\right)$ the same way that we design the feedback gain for the nonlinear feedback control problem. In particular, the compensator gain is given by

$$
L^{T}\left(x_{e}\right)=N^{-1} C \Sigma\left(x_{e}\right)
$$

or equivalently

$$
L\left(x_{e}\right)=\Sigma\left(x_{e}\right) C^{T} N^{-1},
$$

where $\Sigma\left(x_{e}\right)$ satisfies the state estimator dependent Riccati equation

$$
\Sigma\left(x_{e}\right) \hat{A}^{T}\left(x_{e}\right)+\hat{A}\left(x_{e}\right) \Sigma\left(x_{e}\right)-\Sigma\left(x_{e}\right) C^{T} N^{-1} C \Sigma\left(x_{e}\right)+M=0 .
$$

Here $M$ is a symmetric positive semidefinite matrix and $N$ is a symmetric positive definite matrix. They are to be chosen so as to achieve a balance between desired convergence properties and compensator gain. 


\section{State Equations and State Estimator}

The system of differential equations for the state $x$ and the state estimator $x_{e}$ are given by

$$
\begin{aligned}
\dot{x} & =f(x)+B(x) u+w_{s}(t) \\
\dot{x_{e}} & =f\left(x_{e}\right)+B\left(x_{e}\right) u+L\left(x_{e}\right)\left(C x+w_{m}(t)-C x_{e}\right) .
\end{aligned}
$$

The equations for $x$ and $x_{e}$ are indeed coupled due to the term $B(x) u$ in the first equation

of (20) and the term $L\left(x_{e}\right) C x$ in the second equation of (20). Therefore, it is necessary to solve both equations together. In the following we use the notation

$$
\mathbf{x}=\left(\begin{array}{c}
x \\
x_{e}
\end{array}\right) \quad \text { and } \quad \mathbf{G}(\mathbf{x})=\left(\begin{array}{c}
f(x)+B(x) u+w_{s}(t) \\
f\left(x_{e}\right)+B\left(x_{e}\right) u+L\left(x_{e}\right)\left(C x+w_{m}(t)-C x_{e}\right)
\end{array}\right) .
$$

With this notation, the system of differential equations (20) can be expressed as

$$
\dot{\mathbf{x}}=\mathbf{G}(\mathbf{x}) \text {. }
$$

\section{Discretization Method}

We perform the time discretization using backward differentiation formulas (BDF) [15] with a uniform time step $\Delta t$. The solution at time $k \Delta t$ is denoted by $\mathrm{x}^{k}$. Then the discrete form of (20) using BDF formulas is given by

$$
\mathbf{x}^{k+1}=\alpha \Delta t \mathbf{G}\left(\mathbf{x}^{k+1}\right)+\sum_{i=0}^{q-1} \beta_{i} \mathbf{x}^{k-i},
$$

where $q$ is the degree of the $\mathrm{BDF}$ and $\alpha$ and $\beta_{i}$ are constants that depend on $q$. The first-order BDF is the implicit Euler method defined by:

$$
q=1, \quad \alpha=1 \quad \text { and } \quad \beta_{1}=1 .
$$

The second-order BDF is denoted by BDF2 and is given by:

$$
q=2, \quad \alpha=\frac{2}{3}, \quad \beta_{1}=\frac{4}{3} \quad \text { and } \quad \beta_{2}=-\frac{1}{3} .
$$

With the BDF2 method it is necessary to perform the first step of integration using some other numerical method, since it requires the solutions at two previous time steps. A common choice is to use the implicit Euler method for the first time step. It can be shown that this does not reduce the order of accuracy. It is well known that the implicit Euler method and BDF2 have good stability properties [15].

Finally, we note that, at each time step, the implicit Euler method and the BDF2 method require the solution of system of nonlinear equations

$$
\mathbf{x}^{k+1}-\alpha \Delta t \mathbf{G}\left(\mathbf{x}^{k+1}\right)=\sum_{i=0}^{q-1} \beta_{i} \mathbf{x}^{k-i}
$$

for $\mathbf{x}^{k+1}$ in terms of solutions at previous time steps, $\mathbf{x}^{k}, \mathbf{x}^{k-1}$, etc. 


\section{Solution to the Discrete Nonlinear Equations}

The implicit time discretization requires us to solve nonlinear equations of the form

$$
\mathbf{g}(\mathbf{x})=\mathbf{x}-\alpha \Delta t \mathbf{G}(\mathbf{x})-\sum_{i=0}^{p-1} \beta_{i} \mathbf{x}^{k-i}=0 .
$$

In the calculations reported on here, Newton's iterative method [19] was used to obtain the solution of $(21)$. Let the $i$ th iterate be denoted by $\mathbf{x}^{(i)}$. Then Newton's method solves for the $(i+1)$ th iterate in terms of the $i$ th iterate by

$$
\mathbf{x}^{(i+1)}=\mathbf{x}^{(i)}-(\mathbf{I}-\alpha \Delta t \mathbf{J})^{-1} \mathbf{g}\left(\mathbf{x}^{(i)}\right),
$$

where $\mathbf{J}$ is the Jacobian matrix of $\mathbf{G}$.

In the above formula, we need to evaluate $\mathbf{G}$ at $\mathbf{x}$. That is, we must calculate

$$
\mathbf{G}(\mathbf{x})=\left(\begin{array}{c}
A(x) x+a(x)+B(x) u+w_{s}(t) \\
A\left(x_{e}\right) x_{e}+a\left(x_{e}\right)+B\left(x_{e}\right) u+L\left(x_{e}\right)\left(C x+w_{m}(t)-C x_{e}\right)
\end{array}\right),
$$

where the control is given by

$$
u=\min (\max (\tilde{u}, \check{u}), \hat{u})
$$

with

$$
\tilde{u}=-R^{-1} B^{T}\left(x_{e}\right)\left(\Pi\left(x_{e}\right) x_{e}+\tilde{b}\left(x_{e}\right)\right) .
$$

In order to do this we need to solve the state dependent Riccati equation

$$
\Pi\left(x_{e}\right) A\left(x_{e}\right)+A^{T}\left(x_{e}\right) \Pi\left(x_{e}\right)-\Pi\left(x_{e}\right) S\left(x_{e}\right) \Pi\left(x_{e}\right)+Q=0,
$$

where we have used the notation $S\left(x_{e}\right)=B\left(x_{e}\right) R^{-1} B^{T}\left(x_{e}\right)$. In addition, the vector function $\tilde{b}\left(x_{e}\right)$ given by

$$
\tilde{b}\left(x_{e}\right)=\left(A^{T}\left(x_{e}\right)-\Pi\left(x_{e}\right) S\left(x_{e}\right)\right)^{-1}\left(Q y-\Pi\left(x_{e}\right) a\left(x_{e}\right)\right) .
$$

also must be computed. Finally, to evaluate the compensator gain $L\left(x_{e}\right)$, which is given by

$$
L\left(x_{e}\right)=\Sigma\left(x_{e}\right) C^{T} N^{-1}
$$

the dual state dependent Riccati equation

$$
\Sigma\left(x_{e}\right) \hat{A}^{T}\left(x_{e}\right)+\hat{A}\left(x_{e}\right) \Sigma\left(x_{e}\right)-\Sigma\left(x_{e}\right) C^{T} N^{-1} C \Sigma\left(x_{e}\right)+M=0,
$$

with $\hat{A}\left(x_{e}\right)$ given by (18) also must be solved.

To obtain the Jacobian $\mathbf{J}$ of $\mathbf{G}$, one can derive analytical formulas for $\mathbf{J}$ which are rather cumbersome. In practice, it is more convenient to use a finite difference approximation of the Jacobian J. For our application, the forward difference approximation that we employed is sufficiently accurate and of low computational cost.

\section{Simulation Results}

The time period for simulations is 500 days. Unless otherwise stated we have used the time step $\Delta t=1 / 96$, which corresponds to fifteen minutes. The discretization was performed with the second-order backward differentiation formula using the implicit Euler method to compute the first time step. 


\section{$10.1 \quad$ Full State Feedback Control}

We first present simulation results where we assumed that full state observations are available for feedback.

\subsubsection{Optimal treatment in the acute state}

In our first example, we assume that medication starts immediately after HIV infection (an unrealistic assumption in practice-see further comments below). In this case, the initial values for the state variables are given by the acute state

$$
\begin{aligned}
& T_{1}=1000 \frac{\text { cells }}{\mathrm{mm}^{3}}, \quad T_{2}=3.198 \frac{\text { cells }}{\mathrm{mm}^{3}}, \quad T_{1}^{*}=0.0 \frac{\text { cells }}{\mathrm{mm}^{3}}, \quad T_{2}^{*}=0.0 \frac{\text { cells }}{\mathrm{mm}^{3}}, \\
& V_{I}=0.001 \frac{\text { virions }}{\mathrm{mm}^{3}}, \quad V_{N I}=0.0 \frac{\text { virions }}{\mathrm{mm}^{3}}, \quad \text { and } \quad E=0.01 \frac{\text { cells }}{\mathrm{mm}^{3}} .
\end{aligned}
$$

In this example we also assume that the maximum "efficacies" to be $\epsilon_{1}^{\max }=0.7$ and $\epsilon_{2}^{\max }=0.3$.

Figure 1 depicts the dynamics of the suboptimal reverse transcriptase inhibitor (RTI) control $\epsilon_{1}$ and the protease inhibitor (PI) control $\epsilon_{2}$. The corresponding state progressions are shown in Figure 2. After approximately 325 days the state variables have reached near equilibrium and start to oscillate in the neighborhood of the stable "healthy" steady state (4).

After 325 days the feedback control-based treatment calls for many cycles of on and off therapy, each with a short duration of few hours. This type of protocol is difficult for the patients to follow since, for example, many pills need to be taken together with food. Therefore, to avoid this type of treatment schedule in the long term we propose to terminate the medication when the viral load has reached a sufficiently low level. Thus the medication will be administered only when the condition

$$
V_{I}+V_{N I}>1.0 \frac{\text { virions }}{\mathrm{mm}^{3}}
$$

is satisfied. Figure 3 presents the resulting treatment regimen and Figure 4 depicts the corresponding state variables. In this case, even though it does take longer for the state to settle down to the "healthy" equilibrium state, the results confirm that once the system reaches the stable equilibrium state, medication is no longer required. We remark that because of the condition (23) we also do not give any medication during the first two days after the infection, since during this time the viral load is below our limit. In practice, the first treatment would be even further delayed since one would almost never be aware of the infection early on or even when the lower load level in (23) is first exceeded.

So far in our numerical studies discussed above, we used the maximum efficacies $\epsilon_{1}^{\max }=$ 0.7 and $\epsilon_{2}^{\max }=0.3$. An important and interesting question is how low these efficacies can be in order for state of the system to be able to reach the "healthy" equilibrium state. In our previous work [2], the analysis revealed that without any medication the state will converge to the unhealthy steady state (24) given below. Thus, there should be a threshold boundary in the $\left(\epsilon_{1}^{\max }, \epsilon_{2}^{\max }\right)$ efficacy plane which indicates whether it is possible or not to reach the "healthy" equilibrium state with any admissible drug therapy.

We investigated the influence of the maximum efficacies by varying both of them from zero to one and carrying out a series of numerical simulations with the nonlinear feedback 


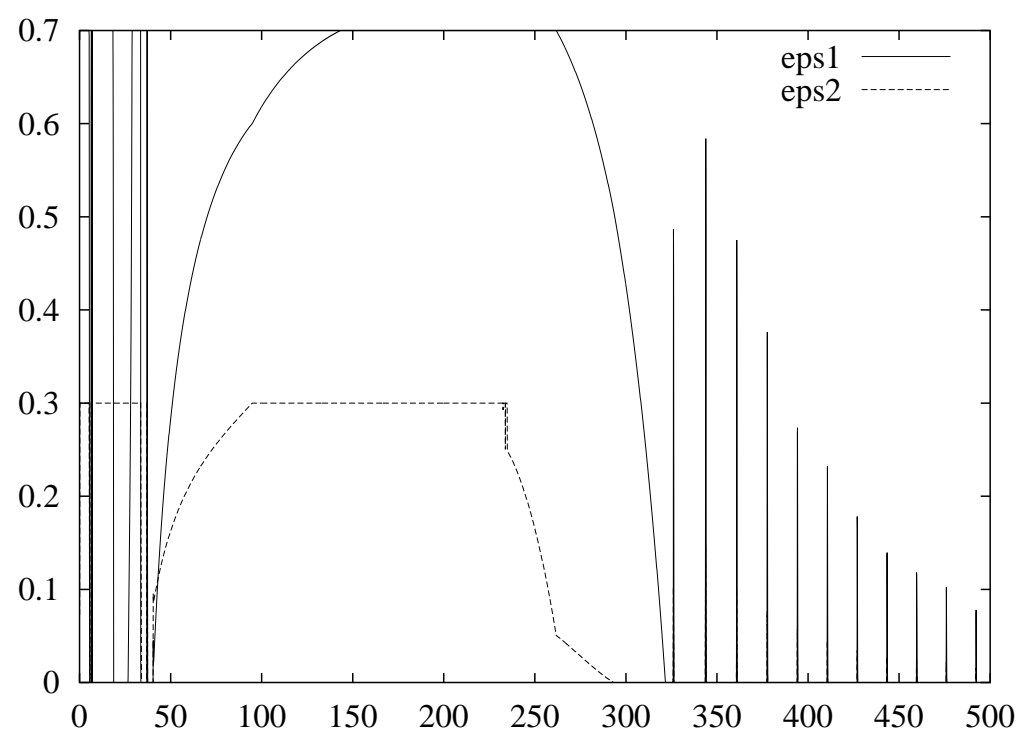

Figure 1: Multidrug therapy starting from the acute state.

controls for each of these combinations of bounds. Figure 5 depicts the immune effector $E$ after 500 days for these simulations. It is noted that in the white region of Figure 5 , the immune effector is around $350 \frac{\text { cells }}{\mathrm{mm}^{3}}$. Hence, for the values of the maximum efficacies in this region the nonlinear suboptimal feedback control will bring the state of the system to the "healthy" equilibrium state.

Finally, from Figure 5 we can observe a slight apparent lack of robustness of the proposed nonlinear feedback control law. This is probably a consequence of the local linearization of the nonlinear dynamics or the suboptimal nature of the approximate nonlinear controller. For example, when the maximum efficacies are $\epsilon_{1}^{\max }=0.8$ and $\epsilon_{2}^{\max }=0.0$, the "healthy" equilibrium state is reachable. However, when the maximum efficacy $\epsilon_{1}^{\max }$ is increased by 0.025 , the "healthy" equilibrium state is no longer reachable.

\subsubsection{Optimal treatment in the unhealthy steady state}

In our second set of examples, we consider a patient who has not taken medication after HIV infection and the disease progression is proceeding towards the unhealthy steady state

$$
\begin{aligned}
& T_{1}=163.573 \frac{\text { cells }}{\mathrm{mm}^{3}}, \quad T_{2}=0.005 \frac{\text { cells }}{\mathrm{mm}^{3}}, \quad T_{1}^{*}=11.945 \frac{\text { cells }}{\mathrm{mm}^{3}}, \quad T_{2}^{*}=0.046 \frac{\text { cells }}{\mathrm{mm}^{3}}, \\
& V_{I}=63.919 \frac{\text { virions }}{\mathrm{mm}^{3}}, \quad V_{N I}=0.0 \frac{\text { virions }}{\mathrm{mm}^{3}}, \quad \text { and } \quad E=0.024 \frac{\text { cells }}{\mathrm{mm}^{3}} .
\end{aligned}
$$

Again the question is whether our suboptimal approximate nonlinear feedback controller can transfer the system from the unhealthy state (24) to the "healthy" equilibrium state (4) in finite time. We begin by letting the maximum efficacies be $\epsilon_{1}^{\max }=0.7$ and $\epsilon_{2}^{\max }=0.3$. The dynamics of the suboptimal treatment strategy proposed by the nonlinear feedback control are shown in Figure 6 and the corresponding state variables are plotted in Figure 7. Clearly, in this case the "healthy" equilibrium state is not reachable. 

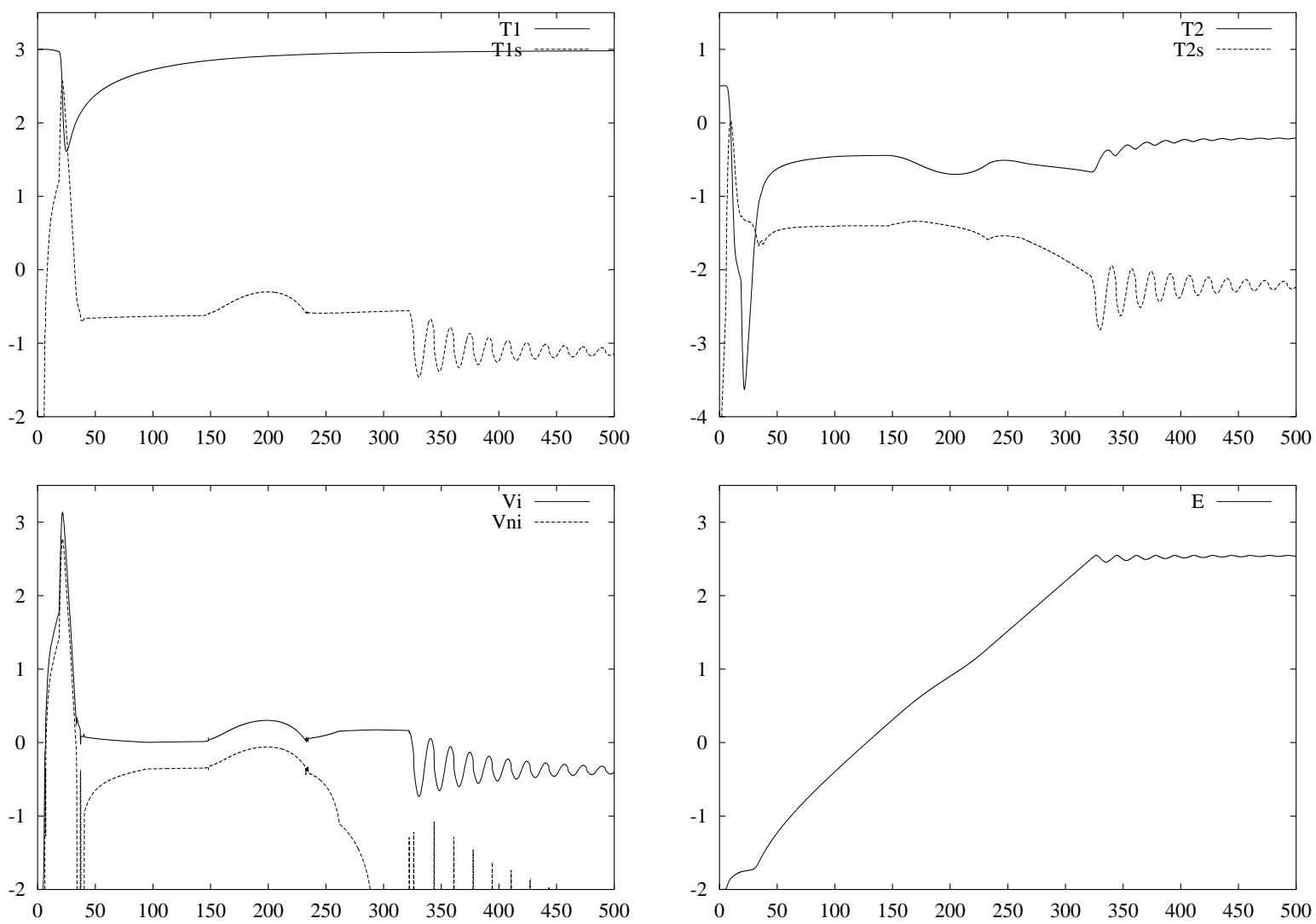

Figure 2: Plots of the state variables with the $y$-axis in $\log _{10}$-scale.

Next we increase the maximum efficacy $\epsilon_{1}^{\max }$ of the reverse transcriptase inhibitor to be 0.75 . The resulting optimal therapy regimen is depicted in Figure 8. The therapy does bring the system to the "healthy" equilibrium state as can be seen in Figure 9. It is noted that we discontinued the medication using the viral load condition (23) as proposed earlier.

As in the earlier example for acute infection, we investigated the combination of the maximum efficacies $\epsilon_{1}^{\max }$ and $\epsilon_{2}^{\max }$ so that "healthy" equilibrium state is reachable. Figure 10 depicts the immune effector $E$ after 500 days as a function of maximum efficacies. We also plotted in Figure 11 contour lines of the so-called "total combined drug efficacy" (some measure of the combined control level)

$$
\epsilon=1-\left(1-\epsilon_{1}\right)\left(1-\epsilon_{2}\right)
$$

as defined in [12]. The threshold boundary in Figure 10 approximates very well the $80 \%$ total efficacy curve given by the equation $\epsilon=0.8$.

Figure 10 demonstrates that slightly smaller maximum efficacies are required than for the acute state in Figure 5. This again suggests a slight lack of robustness in the nonlinear feedback control for the acute state. It is most likely that the approximations are not sufficiently accurate for the highly nonlinear transient period observed after the infection in the acute state case. 


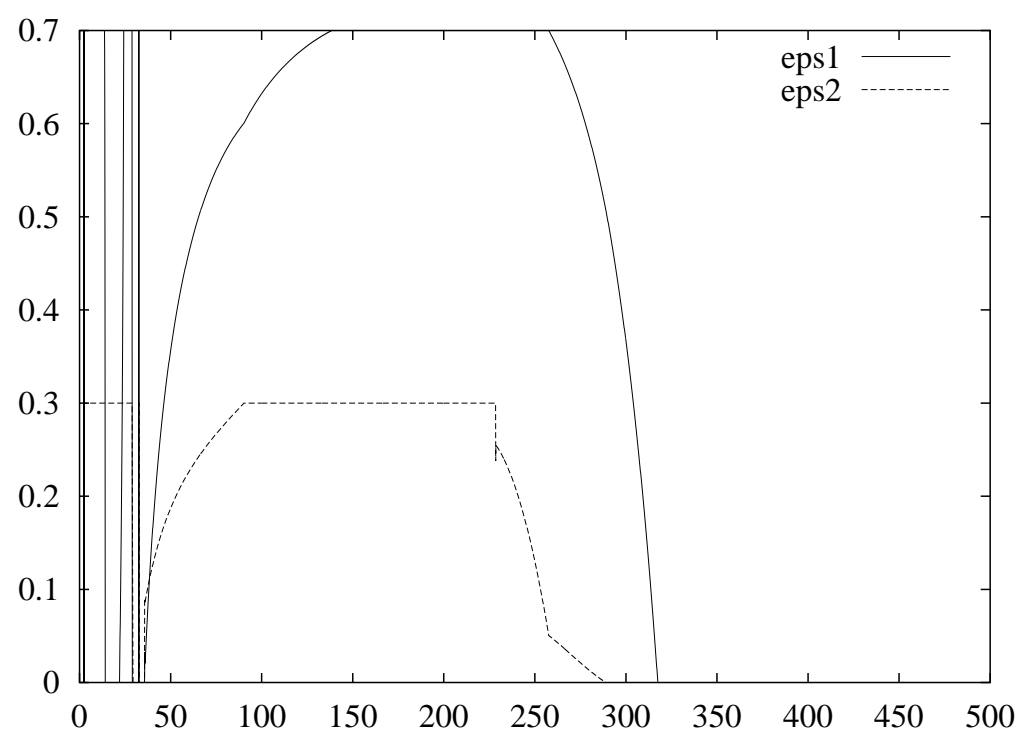

Figure 3: The medication levels starting from the acute state using the stopping criterion based on the viral load.

\subsection{Feedback Control and State Estimation}

In this section, we consider the design and synthesis of nonlinear feedback control-based treatments in which one only has partial state observations.

\subsubsection{Inaccurate initial condition for the state estimator}

We consider first the acute infection case where the initial value for the state $x$ is given by (22). We disturbed the initial value of the state estimator $x_{e}$ by $20 \%$ so that $x_{e}=0.8 x$. As in our earlier computational experiment, the maximum efficacies are $\epsilon_{1}^{\max }=0.7$ and $\epsilon_{2}^{\max }=0.3$. For the computation of the estimator, the weighting matrices in (19) are chosen to be

$$
M=0.01 I_{2 \times 2} \quad \text { and } \quad M=I_{7 \times 7},
$$

where $I_{l, l}$ denotes the $l \times l$ identity matrix. During the 100 first days in the simulation the estimator $x_{e}$ has tendency to have negative components which is not feasible. In such a case, we project the negative components to be zero.

Figure 12 records the suboptimal treatment protocol obtained using the estimator $x_{e}$ in the nonlinear feedback control laws. The therapy dynamics are fairly similar to the ones shown in Figure 3. Figure 13 depicts the Euclidean distance between the state and the estimator. This plot clearly indicates that the state estimator converges to the state asymptotically. In Figure 14 we plot the state variables and their estimators for the first 50 days. After 50 days it is difficult to discern the differences between the state and the estimator in such plots and they are omitted.

\subsubsection{Noisy measurements}

We next consider the suboptimal feedback controller in the presence of noise in the observations. At the $k$ th time step, we denote the measurement without noise and the noise 

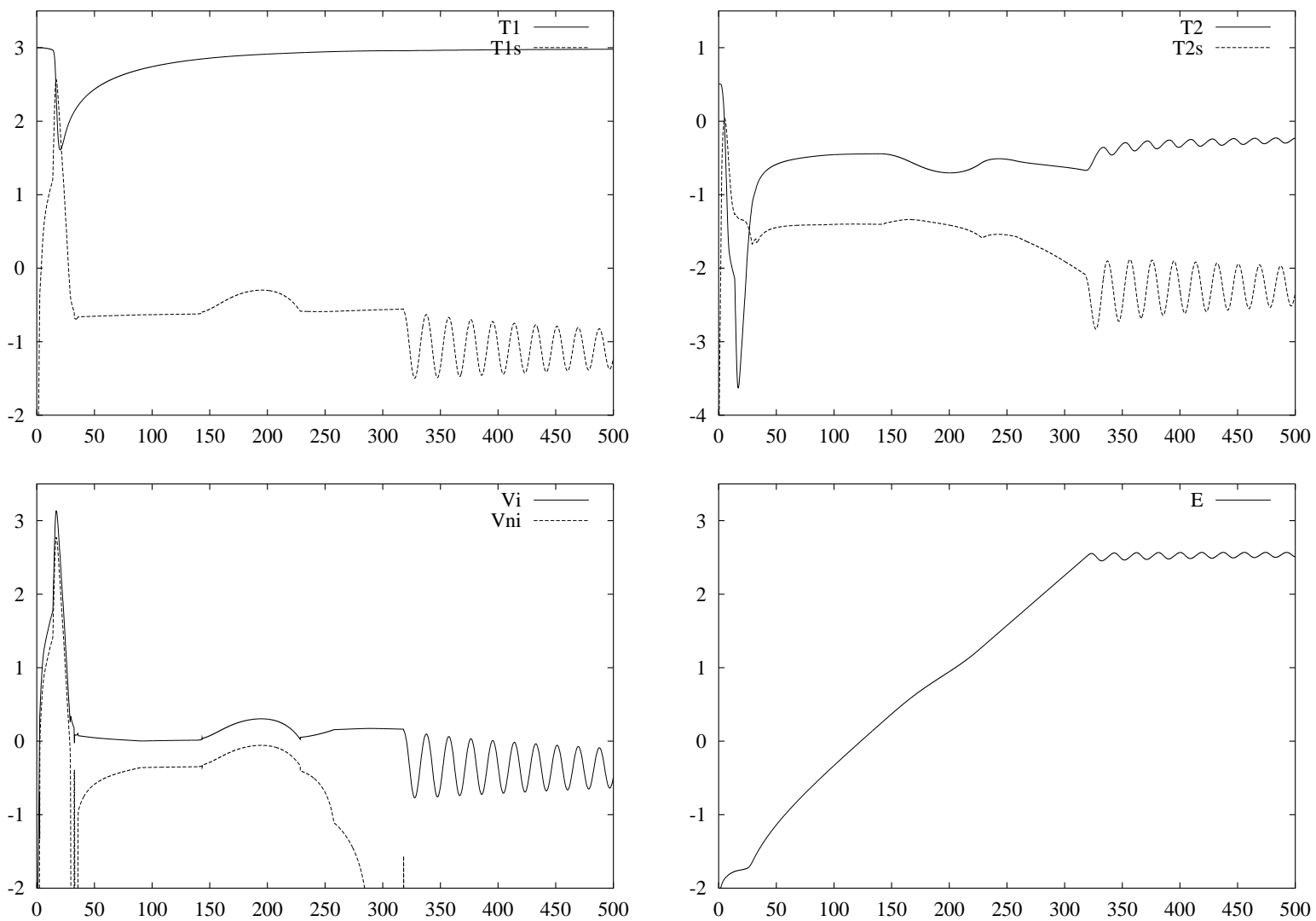

Figure 4: Plots of the state variables using the stopping criterion based on the viral load with the $y$-axis in $\log _{10}$-scale.

by $C x^{k}$ and $w_{m}^{k}$, respectively. We assume the noise to be lognormally distributed without correlation between the components. Furthermore, we assume the noise has zero mean and the variance of the $i$ th component is taken to be $\sigma_{i}^{2}\left(C x^{k}\right)_{i}$. The assays measuring CD4 cell counts and viral loads typically have this type of measurement errors [27, 32]. Under these assumptions the $i$ th component of the noise is given by

$$
\left(w_{m}^{k}\right)_{i}=\exp \left(\mathcal{N}\left(\log \left(C x^{k}\right)_{i}-\frac{1}{2} \log \left(\sigma_{i}^{2}+1\right), \log \left(\sigma_{i}^{2}+1\right)\right)\right)-\left(C x^{k}\right)_{i},
$$

where $\mathcal{N}(\cdot, \cdot)$ is the normal distribution with the mean given by the first argument and the variance given by the second argument. In the following experiment the parameter $\sigma_{i}^{2}$ is chosen as $\sigma_{i}^{2}=(0.10)^{2}$. The measurements without the noise and with the noise are graphed in Figure 15 where the observation sampling time is two hours.

In order to make the estimator $x_{e}$ sufficient for reasonable control, it appears to be necessary to reduce the sampling time increments. For the next results, we used fifteen second sampling time steps, that is, $\Delta t=1 / 5760$. We used the same initial values as in the case without noise, that is, the state corresponds to the acute infection and the state estimator initial value is $20 \%$ off.

Figure 16 depicts the optimal treatment strategy with imperfect measurements. Because of the noise the controls contain some oscillations from 250 days to 320 days. The 


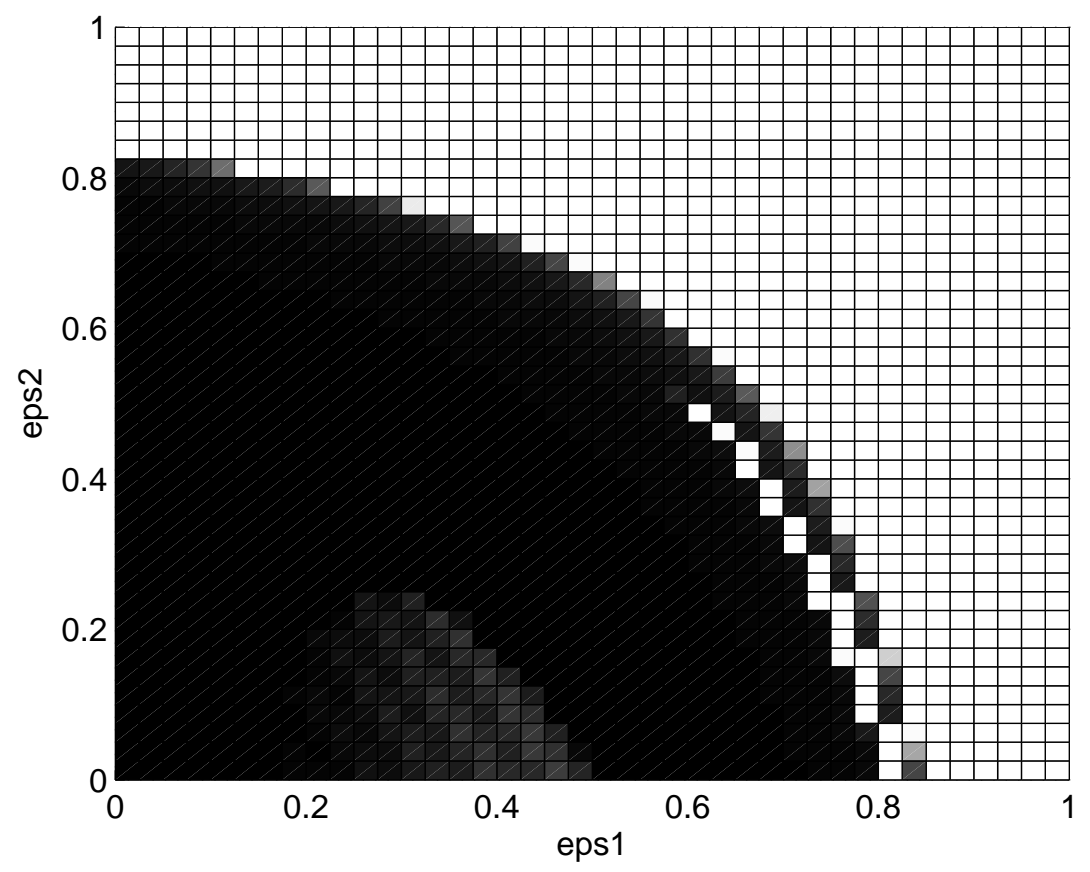

Figure 5: The immune effector $E$ after 500 days after HIV infection for different maximum efficacies $\epsilon_{1}^{\max }$ and $\epsilon_{2}^{\max }$. White and black colors corresponds to values around $370 \frac{\text { cells }}{\mathrm{mm}^{3}}$ and $0.02 \frac{\mathrm{cells}}{\mathrm{mm}^{3}}$, respectively.

short interruptions in the medications during the 143th day and the 305th day are probably due to lack of robustness in our Newton solver and are thus an artifact of our computational schemes. In Figure 17 we plot the state variables and their estimators for the entire 500 days and the Euclidean distance between them. Note that the estimators approximate very well the true states of the system except at the beginning and the end of the simulations. Despite these discrepancies, the suboptimal dynamic multidrug therapy is still very similar to the one without noise in observations depicted in Figure 12. 


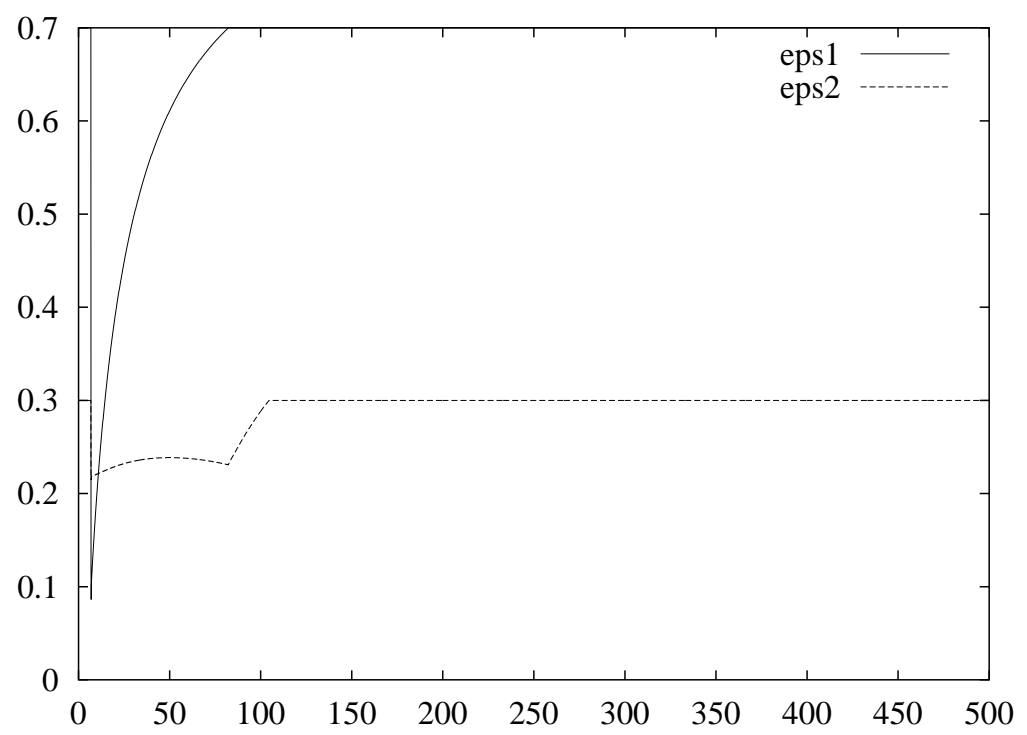

Figure 6: The suboptimal HIV treatment starting from the unhealthy steady state when $\epsilon_{1}^{\max }=0.7$.
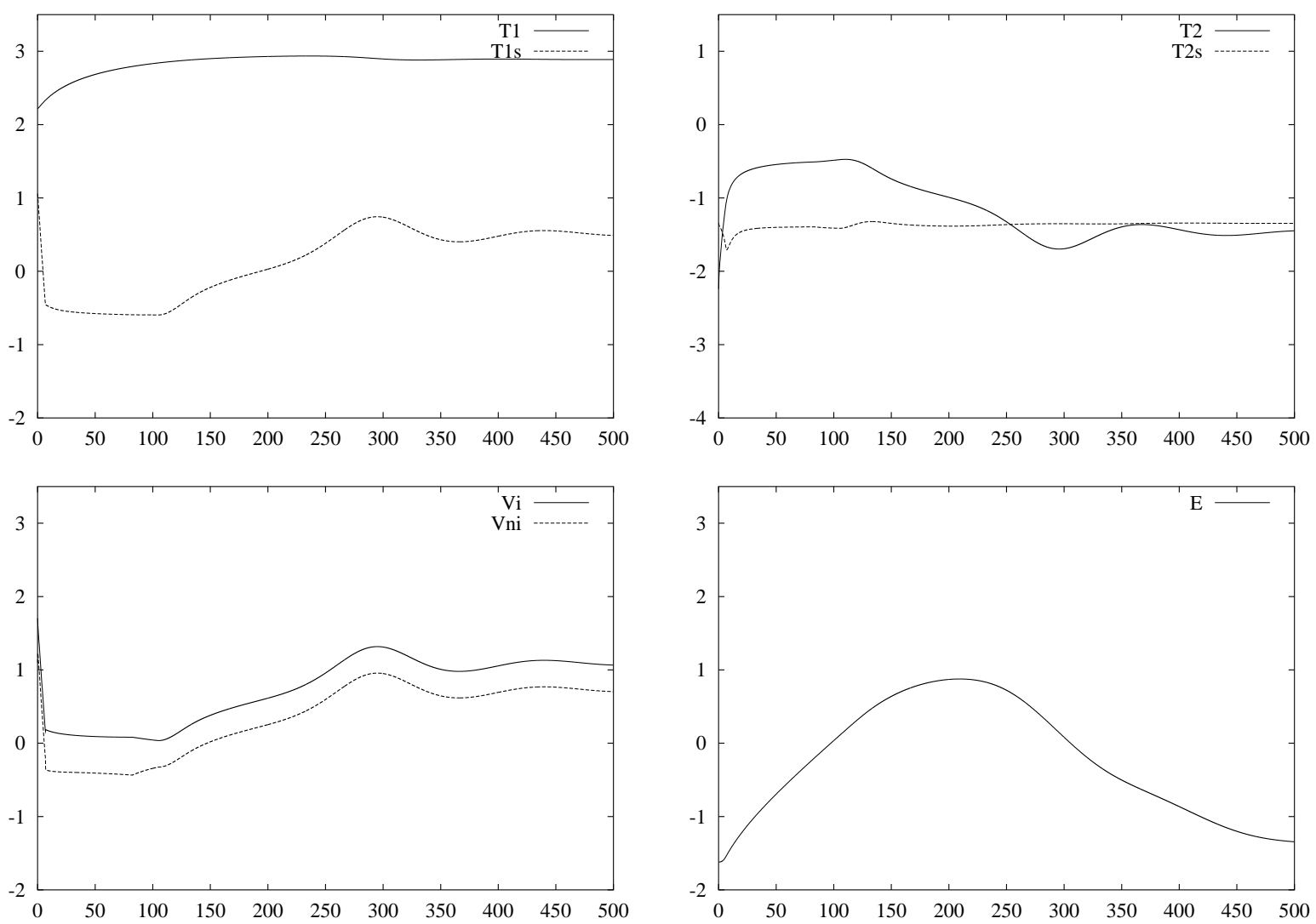

Figure 7: Plots of the state variables with the $y$-axis in $\log _{10}$-scale. 


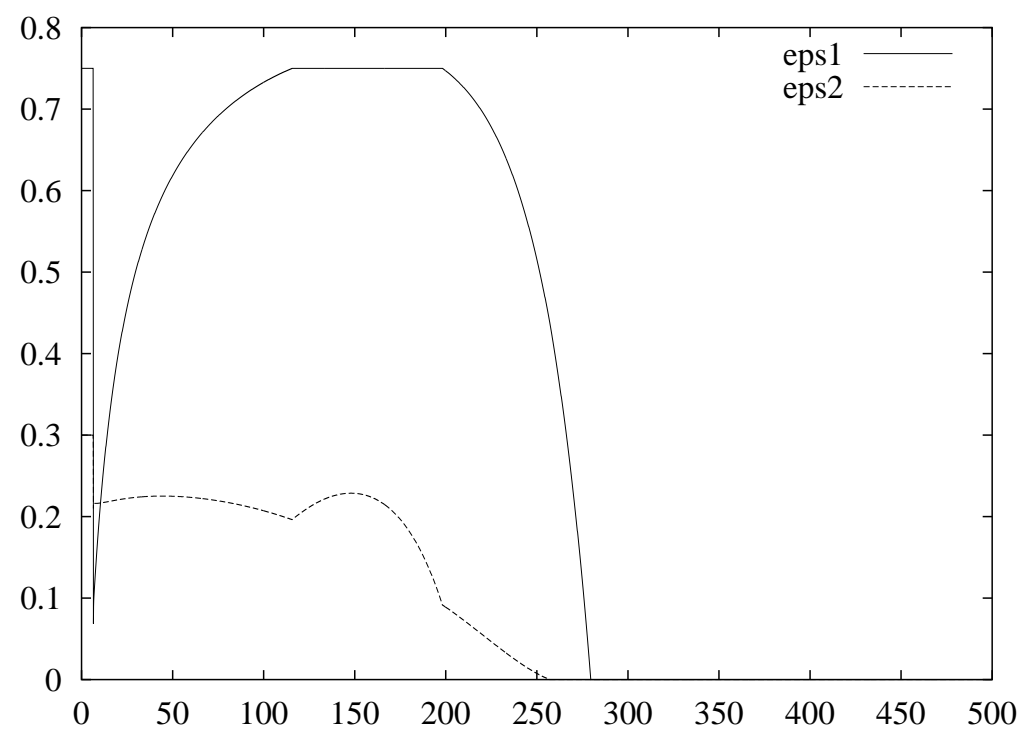

Figure 8: The medication levels starting from the unhealthy steady state when $\epsilon_{1}^{\max }=0.75$.
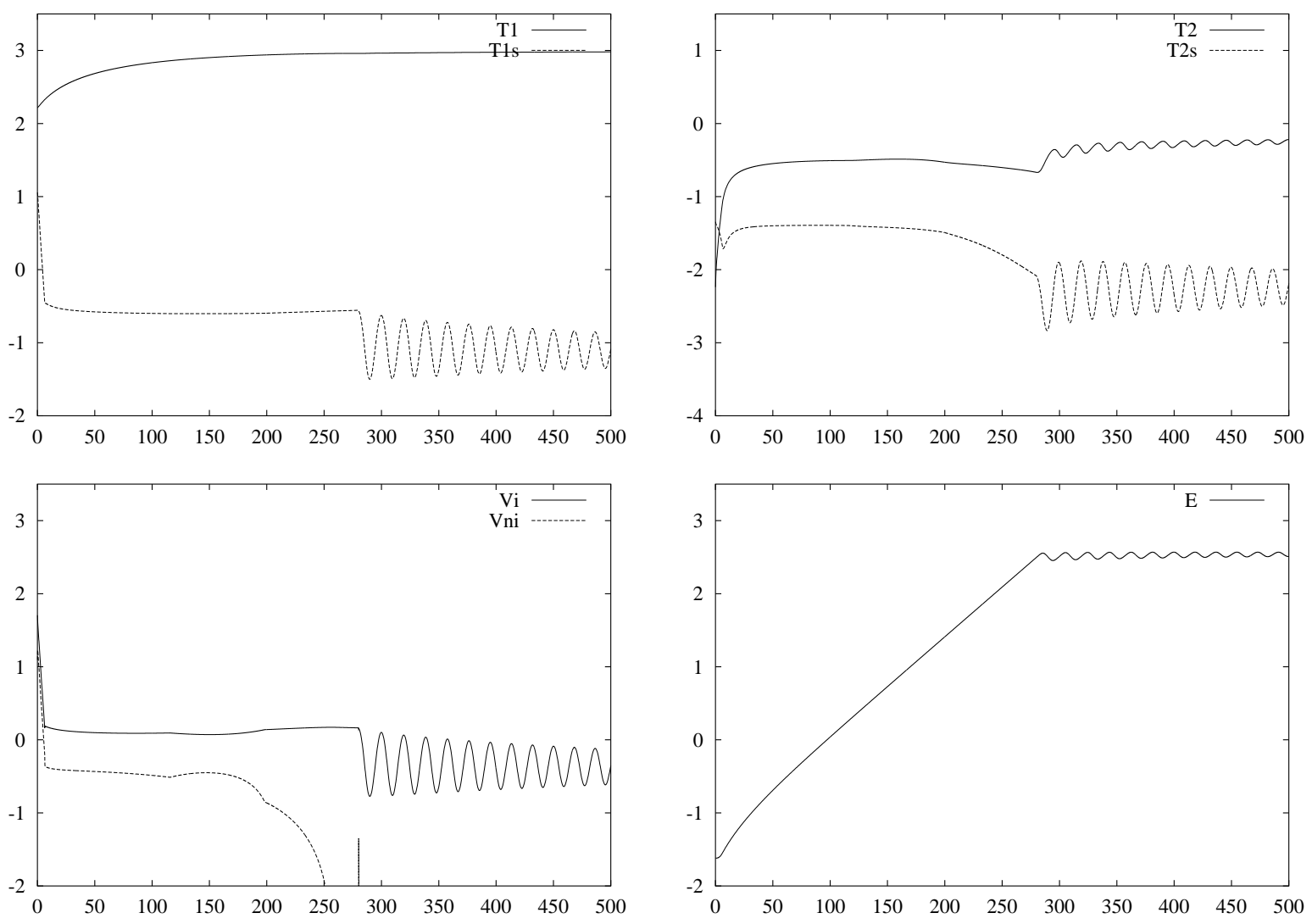

Figure 9: Plots of the state variables with the $y$-axis in $\log _{10}$-scale. 


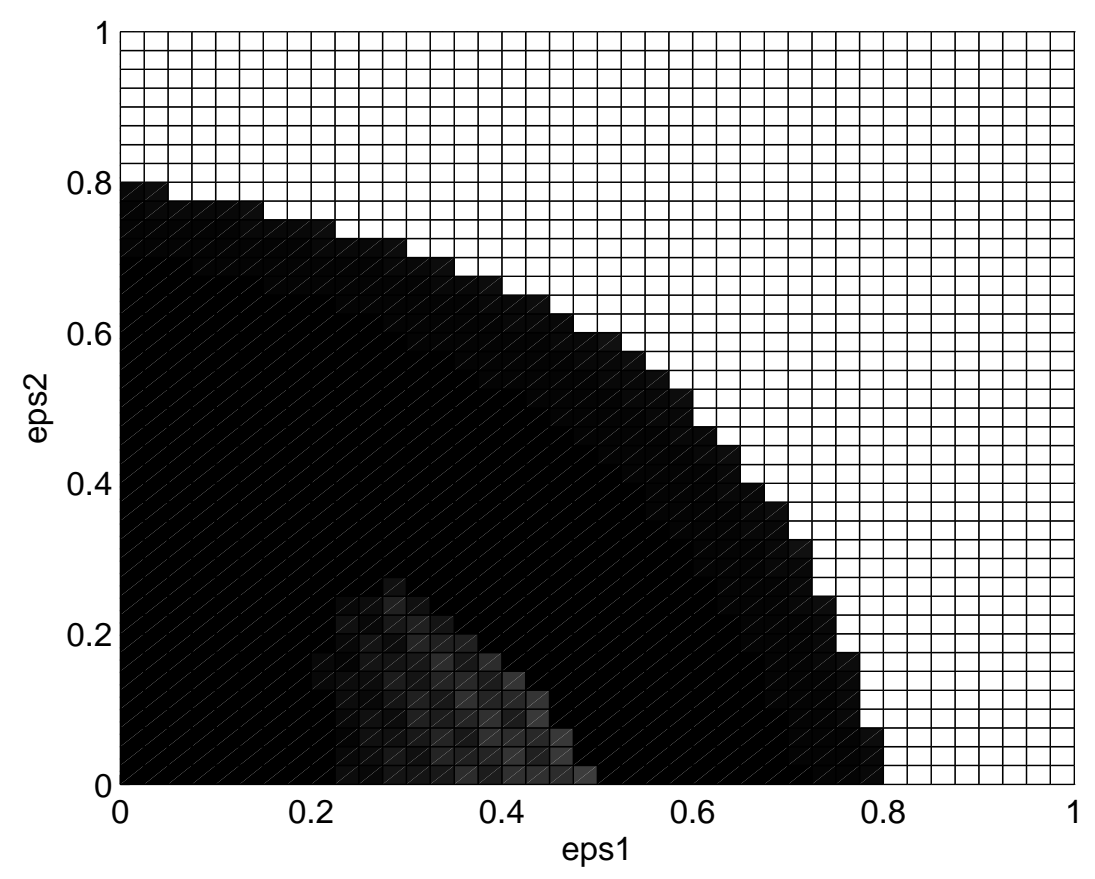

Figure 10: The immune effector $E$ after 500 days after starting medication on the unhealthy state for different maximum efficacies $\epsilon_{1}^{\max }$ and $\epsilon_{2}^{\max }$. White and black colors corresponds to values around $370 \frac{\mathrm{cells}}{\mathrm{mm}^{3}}$ and $0.02 \frac{\text { cells }}{\mathrm{mm}^{3}}$, respectively.

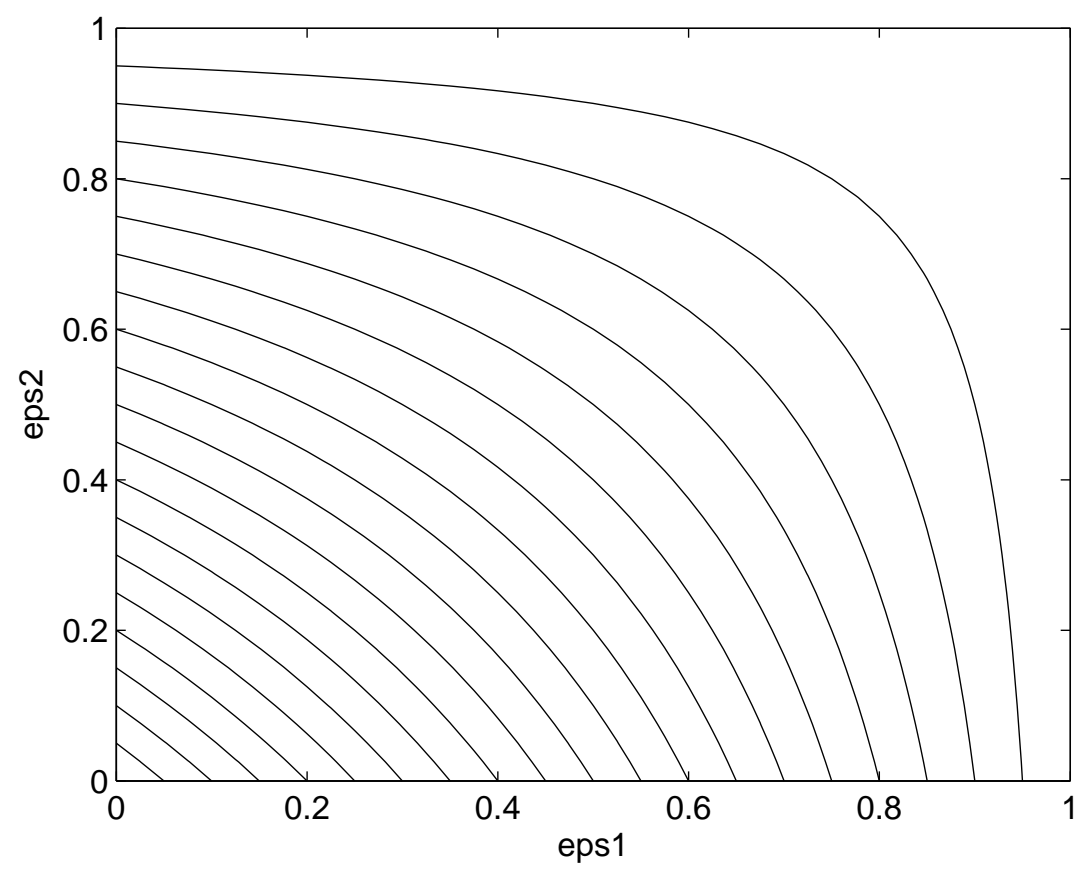

Figure 11: Contour lines of the "total combined drug efficacy" $\epsilon=1-\left(1-\epsilon_{1}\right)\left(1-\epsilon_{2}\right)$. 


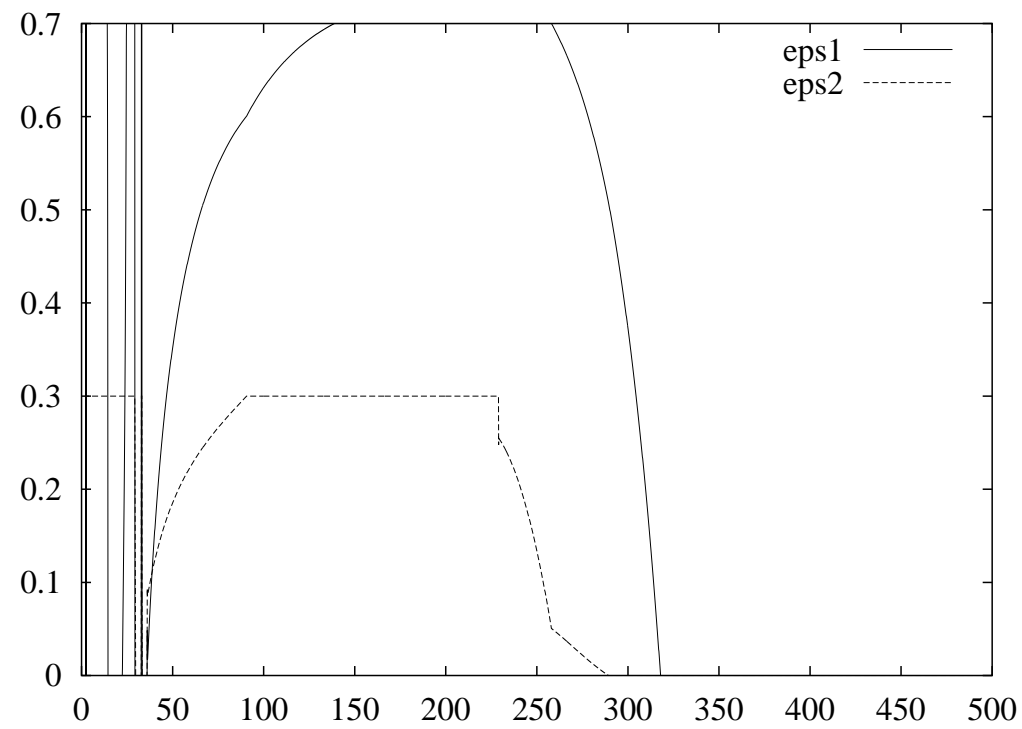

Figure 12: The suboptimal dynamics treatment based on the state estimator.

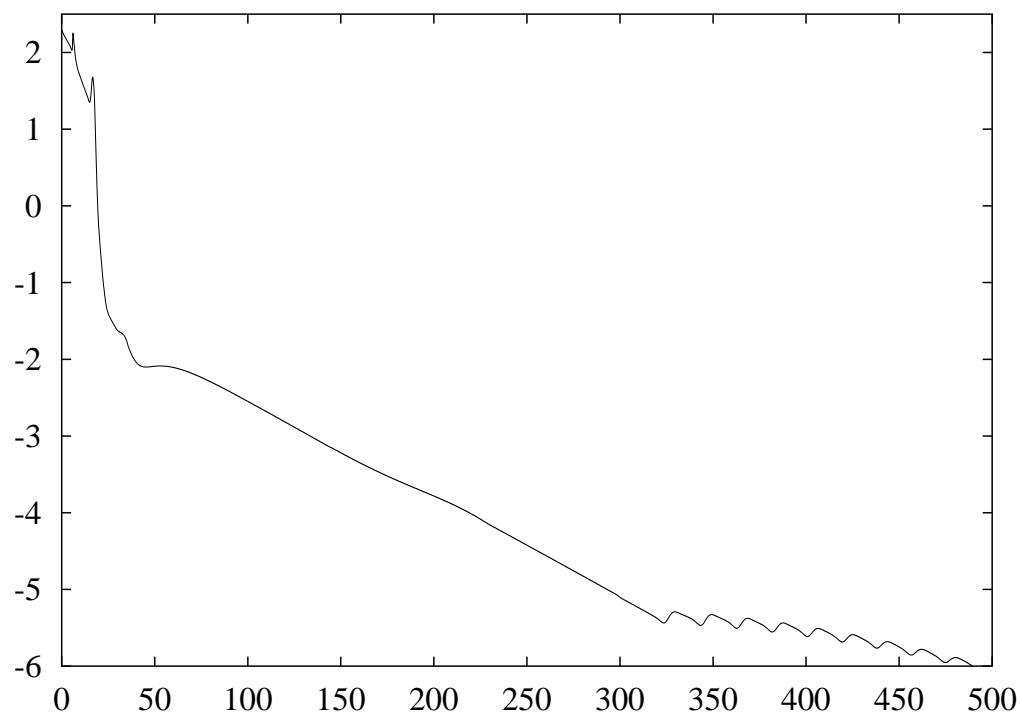

Figure 13: The Euclidean distance between the state and state estimator with the $y$-axis in $\log _{10}$-scale. 

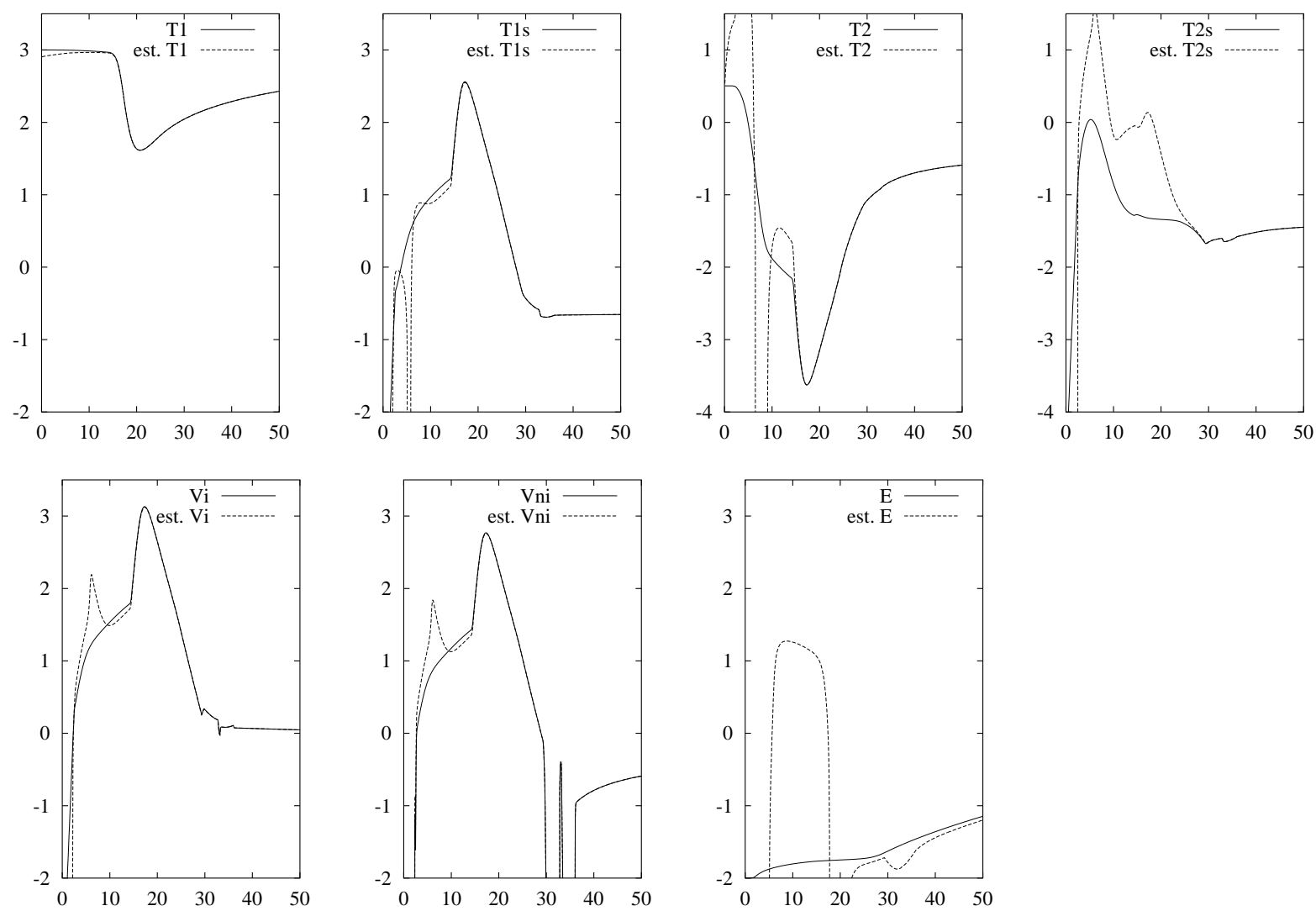

Figure 14: Plots of the state variables and corresponding estimators for the first 50 days with the $y$-axis in $\log _{10}$-scale. 

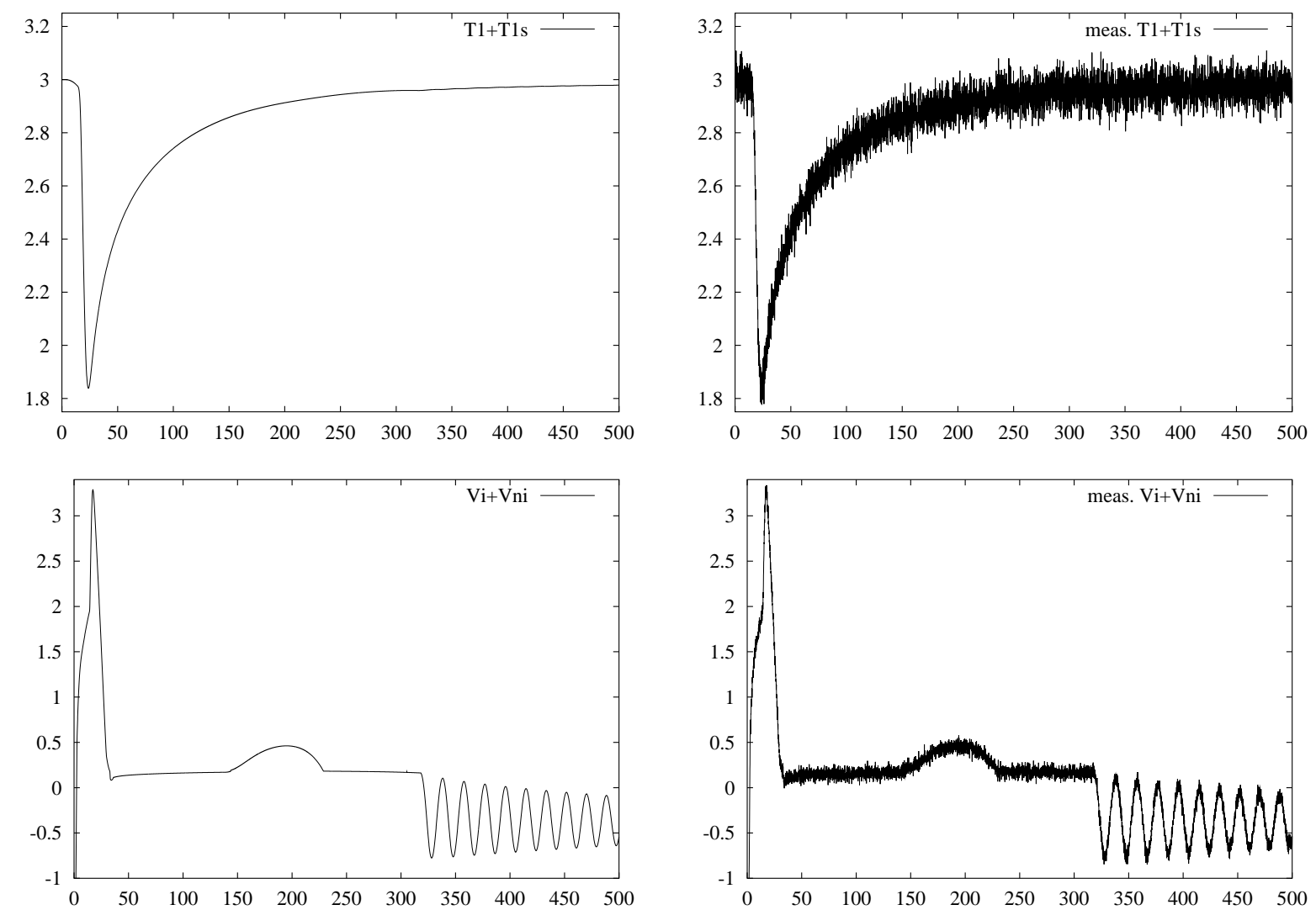

Figure 15: The measurements with the $y$-axis in $\log _{10}$-scale.

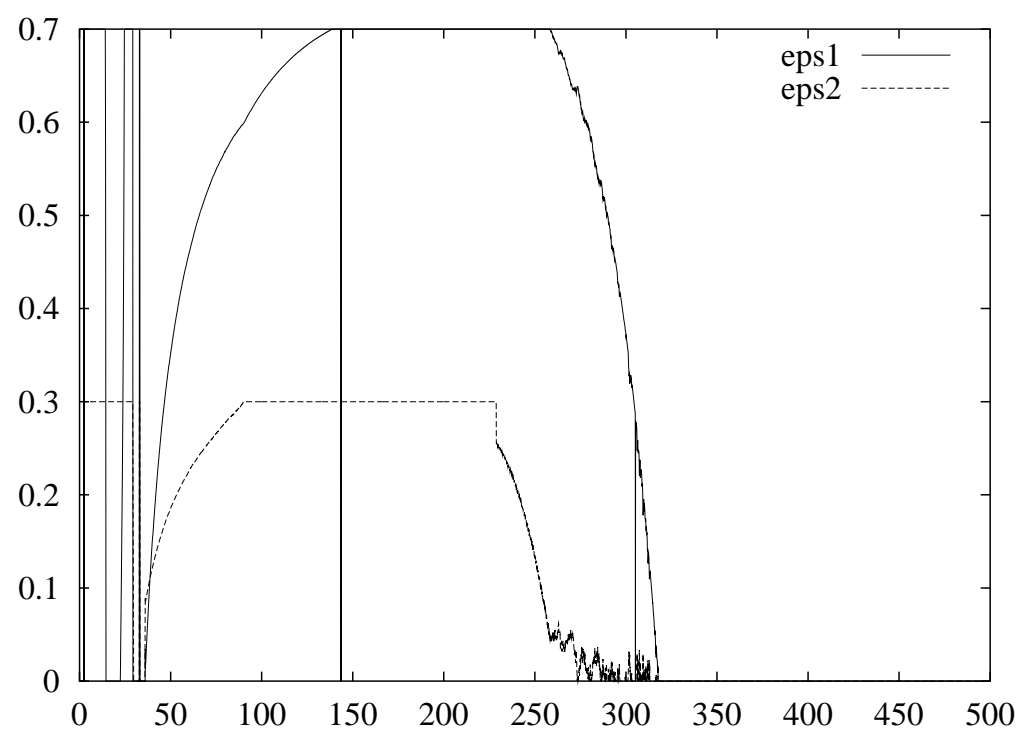

Figure 16: The optimal estimator-based treatment when there is noise in measurements. 

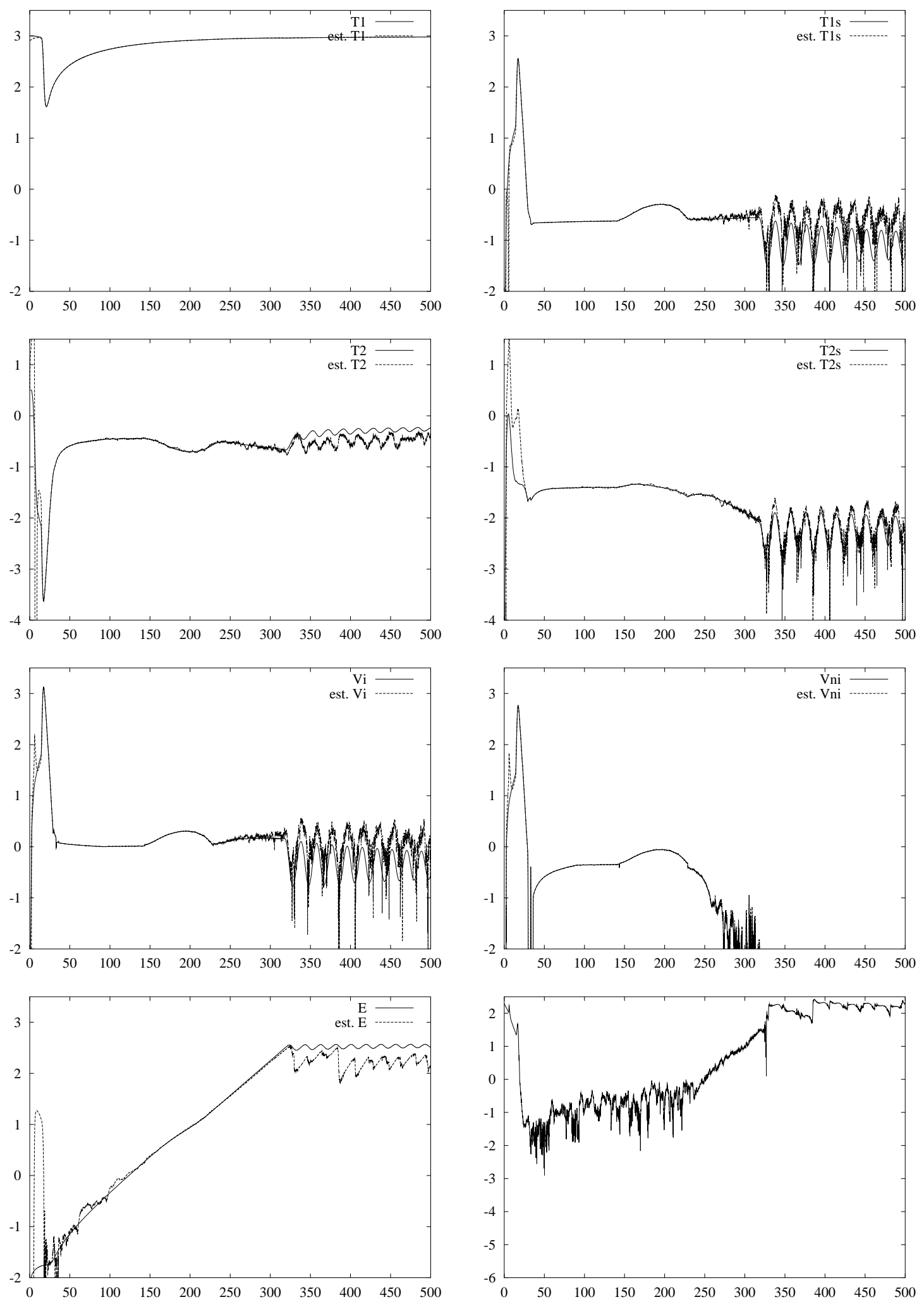

Figure 17: The states, their estimators and the Euclidean distance between them in the lower right corner with the $y$-axis in $\log _{10}$-scale. 


\section{Conclusions}

We presented and used techniques and ideas from control theory to design and synthesize nonlinear feedback control-based treatment regimes for HIV. The mathematical model for HIV progression includes compartments for target cells, infected cells, virus, and immune response that are subjected to multiple (RTI- and PI- type) drug treatments as controllers. We have demonstrated through numerical simulations that by using a "target tracking" approach, suboptimal feedback-based treatment strategies can be designed to move the state of the system from an "unhealthy" state (high virus load and low immune response) to a "healthy" one (with low viral load and high immune effector levels). An important advantage of this drug regimen design is that once the viral load is controlled to very low levels, the drug dosage can be reduced or completely terminated. Consequently, long term pharmaceutical side effects could also be reduced. Thus, this approach suggests that by anticipating and responding to the disease progression, dynamic feedback strategies such as those designed in this paper could lead to long-term control of HIV after discontinuation of therapy.

\section{Acknowledgements}

We are grateful to Brian Adams for a number of constructive comments and suggestions during the course of this work, to Dr. Alan Perelson for stimulating discussions and in particular for his suggestion to include a non infectious virus compartment in the model, and to Dr. Kazufumi Ito for numerous conversations related to feedback control and estimation. This research has been funded in part by the Joint DMS/NIGMS Initiative to Support Research in the Area of Mathematical Biology under grant 1R01GM67299-01 and in part by the US Air Force Office of Scientific Research under grant AFOSR FA 9550-04-1-0220.

\section{References}

[1] B. M. Adams, H. T. Banks, M. Davidian, H.-D. Kwon, H. T. Tran, S. N. Wynne, AND E. S. RosenberG, HIV dynamics: modeling, data analysis, and optimal treatment protocols, J. Comput. Appl. Math., (2005). to appear.

[2] B. M. Adams, H. T. Banks, H.-D. Kwon, and H. T. Tran, Dynamic multidrug therapies for HIV: Optimal and STI control approaches, Math. Biosci. Engrg., 1 (2004), pp. 223-241.

[3] J. Alvarez-Ramirez, M. Meraz, and J. X. Velasco-Hernandez, Feedback control of the chemotherapy of HIV, Intern. J. Bifur. Chaos, 10 (2000), pp. 2207-2219.

[4] S. H. Bajaria, G. Webb, and D. E. Kirschner, Predicting differential responses to structured treatment interruptions during HAART, Bull. Math. Biol., 66 (2004), pp. 1093-1118.

[5] H. T. Banks, S. C. Beeler, G. M. Kepler, and H. T. Tran, Reduced order modeling and control of thin film growth in an HPCVD reactor, SIAM J. Applied Math., 62 (2002), pp. 1251-1280. 
[6] H. T. Banks, B. M. Lewis, And H. T. Tran, Nonlinear feedback controllers and compensators: A state-dependent Riccati equation approach, J. Comp. Optim. and Appl., submitted, CRSC-TR03-26, NCSU, Raleigh, North Carolina (2003).

[7] S. C. Beeler, H. T. Tran, And H. T. Banks, Feedback control methodologies for nonlinear systems, J. Optim. Theory Appl., 107 (2000), pp. 1-33.

[8] S. Bonhoeffer, M. Rembiszewski, G. M. Ortiz, and D. F. Nixon, Risks and benefits of structured antiretroviral drug therapy interruptions in HIV-1 infection, AIDS, 14 (2000), pp. 2313-2322.

[9] M. E. Brandt And G. Chen, Feedback control of a biodynamical model of HIV-1, IEEE Trans. Biom. Engrg., 48 (2001), pp. 754-759.

[10] S. Butler, D. Kirschner, And S. Lenhart, Optimal control of chemotherapy affecting the infectivity of HIV, in Advances in Mathematical Population Dynamics: Molecules, Cells, Man, O. Arino, D. Axelrod, M. Kimmel, and M. Langlais, eds., World Scientific Publishing, 1997, pp. 104-120.

[11] M. A. L. Caetano and T. Yoneyama, Short and long period optimization of drug doses in the treatment of AIDS, An. Acad. Bras. Ciênc., 74 (2002), pp. 379-392.

[12] D. S. Callaway and A. S. Perelson, HIV-1 infection and low steady state viral loads, Bull. Math. Biol., 64 (2002), pp. 29-64.

[13] R. Culshaw, S. Ruan, and R. J. Spiteri, Optimal HIV treatment by maximising immune response, J. Math. Biol., 48 (2004), pp. 545-562.

[14] K. R. Fister, S. Lenhart, and J. S. McNally, Optimizing chemotherapy in an HIV model, Electron. J. Differential Equations, 1998 (1998), pp. 1-12.

[15] E. Hairer and G. Wanner, Solving ordinary differential equations. II. Stiff and differential-algebraic problems, vol. 14 of Springer Series in Computational Mathematics, Springer-Verlag, Berlin, second ed., 1996.

[16] R. A. Hull, J. R. Cloutier, C. P. Mracek, and D. T. Stansbery, Statedependent Riccati equation solution of the toy nonlinear optimal control problem, in Proceedings of the 1998 American Control Conference, vol. 3, 1998, pp. 1658-1662.

[17] A. M. Jeffrey, X. Xia, And I. K. Craig, When to initiate HIV therapy: A control theoretic approach, IEEE Trans. Biom. Engrg., 50 (2003), pp. 1213-1220.

[18] H. R. Joshi, Optimal control of an HIV immunology model, Optimal Control Appl. Methods, 23 (2002), pp. 199-213.

[19] C. T. Kelley, Solving nonlinear equations with Newton's method, vol. 1 of Fundamentals of Algorithms, Society for Industrial and Applied Mathematics (SIAM), Philadelphia, PA, 2003.

[20] D. Kirschner, S. Lenhart, and S. Serbin, Optimal control of the chemotherapy of HIV, J. Math. Biol., 35 (1997), pp. 775-792. 
[21] J. J. Kutch And P. Gurfil, Optimal control of HIV infection with a continuouslymutating viral population, in Proceedings of the 2002 American Control Conference, vol. 5, 2002, pp. 4033-4038.

[22] U. Ledzewicz AND H. SchätTleR, On optimal controls for a general mathematical model for chemotherapy of HIV, in Proceedings of the 2002 American Control Conference, vol. 5, 2002, pp. 3454-3459.

[23] C. P. Mracek and J. R. Cloutier, Control designs for the nonlinear benchmark problem via the state-dependent Riccati equation method, Intern. J. Robust Nonlinear Control, 8 (1998), pp. 401-433.

[24] A. S. Perelson and P. W. Nelson, Mathematical analysis of HIV-1 dynamics in vivo, SIAM Rev., 41 (1999), pp. 3-44.

[25] A. Perelson, D. Kirschner, and R. De Boer, The dynamics of HIV infection of CD4 + T cells, Math. Biosci., 114 (1993), pp. 81-125.

[26] H. Shim, S. J. Han, C. C. Chung, S. Nam, and J. H. Seo, Optimal scheduling of drug treatment for HIV infection: Continuous dose control and receding horizon control, Intern. J. Control Autom. Systems, 1 (2003), pp. 401-407.

[27] A. A. Tsiatis, V. DeGruttola, and M. S. Wulfsohn, Modeling the realitionship of survival to longitudinal data measured with error. applications to survival and CD4 counts in patients with aids, J. Amer. Stat. Assoc., 90 (1995), pp. 27-37.

[28] L. M. Wein, S. A. Zenios, and M. A. Nowak, Dynamic multidrug therapies for HIV: A control theoretic approach, J. Theor. Biol., 185 (1997), pp. 15-29.

[29] D. Wodarz And M. A. Nowak, Specific therapy regimes could lead to long-term immunological control of HIV, PNAS, 96 (1999), pp. 14464-14469.

[30] — Mathematical models of HIV pathogenesis and treatment, BioEssays, 24 (2002), pp. $1178-1187$.

[31] D. Wodarz, Helper-dependent vs. helper-independent CTL responses in HIV infection: Implications for drug therapy and resistance, J. Theor. Biol., 213 (2001), pp. 447459.

[32] H. Wu And A. A. Ding, Population HIV-1 dynamics in vivo: Applicable methods and inferential tools for virological data from AIDS clinical trials, Biometrics, 55 (1999), pp. $410-418$.

[33] R. Zurakowski, M. J. Messina, S. E. Tuna, and A. R. Teel, HIV treatment scheduling via robust nonlinear model predictive control, in Proceedings of the 5th Asian Control Conference, 2004.

[34] R. Zurakowski, A. R. Teel, And D. Wodarz, Utilizing alternate target cells in treating HIV infection through scheduled treatment interruptions, in Proceedings of the 2004 Ameican Control Conference, vol. 1, 2004, pp. 946-951. 
[35] R. Zurakowski and A. R. Teel, Enhancing immune response to HIV infection using MPC-based treatment scheduling, in Proceedings of the 2003 American Control Conference, vol. 2, 2003, pp. 1182-1187. 